\title{
The local amplification of surface waves: A new observable to constrain elastic velocities, density, and anelastic attenuation
}

\author{
Fan-Chi Lin, ${ }^{1}$ Victor C. Tsai, ${ }^{1}$ and Michael H. Ritzwoller ${ }^{2}$ \\ Received 7 February 2012; revised 19 April 2012; accepted 22 April 2012; published 5 June 2012.
}

[1] The deployment of USArray across the continental U.S. has prompted developments within surface wave tomography to exploit this unprecedented data set. Here, we present a method to measure a new surface wave observable: broadband surface wave amplification that provides new and unique constraints on elastic velocities and density within the crust and upper mantle. The method, similar to its phase velocity counterpart referred to as Helmholtz tomography, initiates by constructing phase travel time and amplitude maps across the array for each period and earthquake. Spatial differential operators are then applied to evaluate the amplitude variation, as well as the effect of focusing/defocusing. Based on the 2-D damped wave equation, the amplitude variation corrected for focusing/ defocusing is linked directly to both local amplification and intrinsic attenuation, which are separated by examining waves propagating in opposite directions. We apply the method to teleseismic Rayleigh waves observed across USArray between periods of 24 and $100 \mathrm{~s}$ and show that the observed amplification maps are strongly correlated with known geological features. Small-scale attenuation measurements are contaminated by wavefield complexities, but larger-scale anelastic attenuation is estimated reliably. The observed amplification maps compare well with predictions based on recent 3-D shear velocity models of the western U.S. that were produced from ambient noise and earthquake data. Notably, predictions based on models with different prescribed density structures demonstrate the potential for using estimates of local amplification to constrain not only 3-D velocity structure but also density.

Citation: Lin, F.-C., V. C. Tsai, and M. H. Ritzwoller (2012), The local amplification of surface waves: A new observable to constrain elastic velocities, density, and anelastic attenuation, J. Geophys. Res., 117, B06302, doi:10.1029/2012JB009208.

\section{Introduction}

[2] Surface waves, which propagate near the earth's surface, are sensitive to the elastic, anelastic, and density structure of the crust and upper mantle. Most surface wave tomography studies investigate shear velocity structure by measuring the group and phase travel times of surface waves [e.g., Trampert and Woodhouse, 1996; Ekström et al., 1997; Shapiro et al., 2005; Lin et al., 2011a]. Due to the complexity of source, path, and receiver effects, studies based on amplitude measurements to infer velocity structure [e.g., Dalton and Ekström, 2006a; Yang and Forsyth, 2006a; Pollitz and Snoke, 2010; Lin and Ritzwoller, 2011a] and anelastic structure [e.g., Dalton and Ekström, 2006b; Yang

\footnotetext{
${ }^{1}$ Seismological Laboratory, Division of Geological and Planetary Sciences, California Institute of Technology, Pasadena, California, USA.

${ }^{2}$ Center for Imaging the Earth's Interior, Department of Physics, University of Colorado Boulder, Boulder, Colorado, USA.

Corresponding author: F.-C. Lin, Seismological Laboratory, Division of Geological and Planetary Sciences, California Institute of Technology, 1200 E. California Blvd., MS 252-21, Pasadena, CA 91125, USA. (linf@caltech.edu)

Copyright 2012 by the American Geophysical Union. 0148-0227/12/2012JB009208
}

and Forsyth, 2008; Lawrence and Prieto, 2011; Lin et al., $2011 \mathrm{~b}$ ] are relatively less common. One of the reasons that such studies are less common is that seismometers are usually more poorly calibrated in amplitude than in phase. Although surface waves are sensitive to density structure within the crust and uppermost mantle, the estimation of density is typically considered to be too difficult using surface waves information alone [Tanimoto, 1991]. Instead, some authors have approached the problem by jointly interpreting surface wave dispersion measurements along with gravity [e.g., Maceira and Ammon, 2009]. Estimation of the distribution of density and anelastic attenuation in 3-D is highly desirable, however, because this information would place new constraints on in situ temperature and composition and, perhaps even more intriguingly, would present a means to evaluate fundamental geodynamic driving forces.

[3] The recent deployment of the USArray Transportable Array (Figure 1a) across the U.S. permits the study of the broadband surface wave wavefield in greater detail than ever possible before on continental scales [e.g., Pollitz, 2008; Liang and Langston, 2009]. By taking advantage of the 2-D array configuration and the approximately $2-\mathrm{D}$ nature of surface wave propagation [Tanimoto, 1990; Wielandt, 1993; Tromp and Dahlen, 1993], two new tomographic methods referred to as eikonal and Helmholtz tomography have been 
(a)

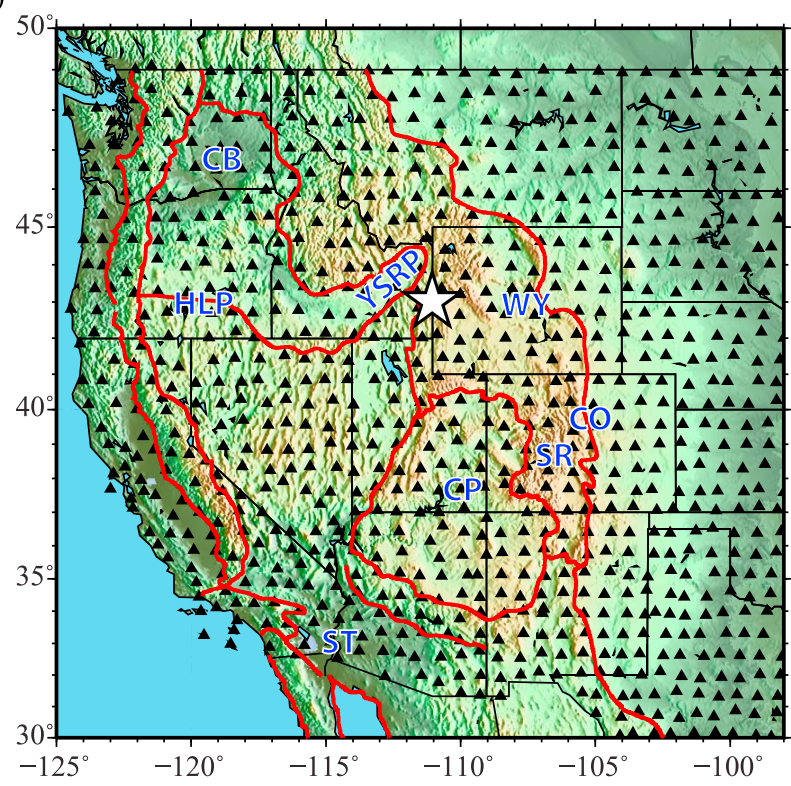

(b)

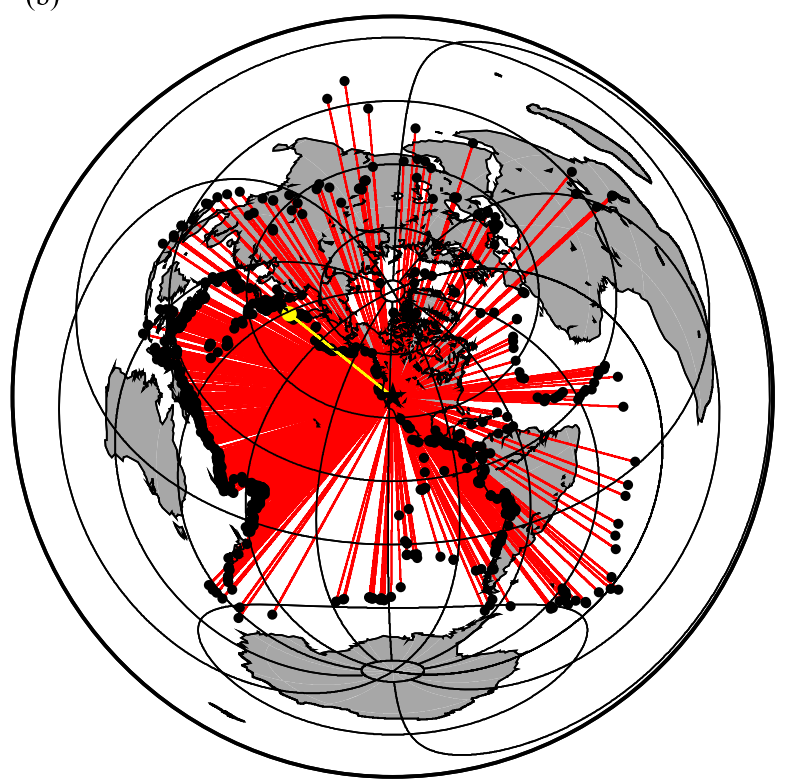

Figure 1. (a) Triangles identify the USArray stations used in this study. The red lines are tectonic and other boundaries in the western U.S. CB: Columbia Basin; ST: Salton Trough; YSRP: Yellowstone/Snake River Plain; CP: Colorado Plateau; WY: Wyoming; SR: Southern Rockies; CO: Colorado; HLP: High Lava Plains. The star marks the location shown in Figures 6a, 6b, and A4a. (b) The earthquakes used in this study. Dots mark the location of the earthquakes, the star is the center of our study region, and the red lines between dots and the star are great circle paths. The yellow circle and path mark the earthquake used in Figures 2 and 3.

developed by Lin et al. [2009] and Lin and Ritzwoller [2011a]. The methods estimate directionally dependent phase velocity by locally applying the $2-\mathrm{D}$ wave equation based on phase travel time and amplitude measurements. The methods start by empirically constructing phase travel time and amplitude maps for surface waves emitted by an earthquake or observed on interstation cross correlations for ambient noise [Lin et al., 2009]. Spatial differential operators (the gradient and Laplacian) are then applied to the travel time and amplitude fields to estimate the apparent velocity and corrections for finite-frequency effects. This type of local inversion method mitigates the need to model the source mechanism and structure outside of the array by focusing explicitly on local wave propagation [Pollitz and Snoke, 2010]. The methods have been applied successfully to USArray data to resolve high-resolution 3-D isotropic and anisotropic structures in the crust and upper mantle across the western U.S. [Moschetti et al., 2010a, 2010b; Lin et al., 2011a].

[4] In the present study, we extend these tomographic methods to study the local amplification and anelastic attenuation of surface waves, which potentially provides independent new constraints on the elastic velocities, density, and anelastic structure of the crust and upper mantle compared to traditional phase and group velocity tomography. The local amplification, sometimes also referred to as the receiver term or local site response [e.g., Dalton and Ekström, 2006b; Yang and Forsyth, 2006a; Levshin et al., 2010], is a measure of the effect of local velocity and density structure beneath the receiver on the observed surface wave amplitude. The fundamental physical principle underlying local amplification is conservation of energy flux, where a wave traveling from a denser/faster medium (high impedance) to a lighter/slower medium (low impedance) will undergo an increase in amplitude. Similarly, a wave traveling from a lighter/slower medium (low impedance) to a denser/faster medium (high impedance) will experience an amplitude reduction. Theoretical studies [e.g., Tromp and Dahlen, 1992] have shown that for a smoothly varying earth model, where modal coupling can be ignored, the local amplification can be determined if the 1-D velocity and density profiles are known directly beneath the location (see section 2). Conversely, the ability to measure the local amplification would provide a direct constraint on the local velocity and density structures. The simultaneous local measurement of surface wave velocity and amplification, therefore, provides a hitherto unexploited constraint on density. Moreover, the ability to constrain local amplification would also reduce bias in estimates of anelastic attenuation. Analogous to studies based on the dispersion of phase and group velocities, the vertical distribution of density may, in principle, be inferred by investigating the frequency dependence of surface wave amplification.

[5] Because local amplification only affects the amplitude measurement at the station location, traditional surface wave tomography studies [e.g., Dalton and Ekström, 2006b] based on a sparse station distribution could not resolve spatial variations in local amplification. The existence of regional arrays such as USArray, on the other hand, allows the amplification to be observed and inverted at the station locations together with other surface wave parameters [e.g., Yang and Forsyth, 2006a]. To estimate the local amplification 
accurately, the effects of both elastic focusing/defocusing and anelastic attenuation must be accounted for properly in the surface wave amplitude measurements [Dalton and Ekström, 2006b; Levshin et al., 2010]. The situation is further complicated by the practically indistinguishable effects of local amplification and variations in instrument responses. To our knowledge, local amplification has not yet been used to study 3-D velocity and density structure.

[6] Anelastic attenuation, on the other hand, describes the intrinsic decay of wave energy lost to heat. The ability to estimate intrinsic anelastic attenuation at relatively high resolution may improve understanding of the temperature state of earth's interior [e.g., Jackson et al., 2002]. Detailed source, path, and receiver effects need to be accounted for to constrain anelastic attenuation accurately based on surface waves [e.g., Dalton and Ekström, 2006b]. While source effects can potentially be mitigated by studying regional wave propagation [e.g., Yang and Forsyth, 2006a], as in this study, detailed path and receiver effects such as focusing/ defocusing and location amplification are also extremely important. Recently, interest has been growing in the use of ambient noise cross correlations to estimate crustal attenuation [e.g., Prieto et al., 2009; Lawrence and Prieto, 2011; Lin et al., 2011b; Tsai, 2011]. At high frequencies, however, the effects of elastic scattering are very important and distinguishing scattering attenuation from anelastic attenuation is challenging.

[7] In this study, we show that high-resolution local amplification and large-scale anelastic attenuation maps can be reliably constructed empirically by examining the surface wave amplitude and phase travel time measurements across USArray based on earthquake data. In particular, similar to eikonal and Helmholtz tomography [Lin et al., 2009; Lin and Ritzwoller, 2011a], we apply spatial differential operators to the observed amplitude and phase travel time fields to determine the effects of focusing/defocusing, attenuation, and local amplification. We use the Laplacian of phase travel time, which essentially describes phase front curvature, to account for the effects of focusing/defocusing. To distinguish the effects of attenuation from amplification, measurements for waves coming from different directions are compared. To validate the observed amplification maps, we show that the maps are correlated with known velocity structures and generally agree well with predictions based on realistic 3-D velocity models constrained by phase velocity measurements alone. We suggest that several detailed discrepancies between the predicted and observed amplification maps are at least partially due to inaccuracies in the density model used. While the observed high-resolution attenuation maps are probably biased by unmodeled surface wave phenomena near sharp structural boundaries, large-scale attenuation maps appear to be well correlated with known tectonic features. More specifically, the tectonically active western U.S. and the eastern stable cratonic U.S. are, as expected, characterized by high and low anelastic attenuation, respectively.

[8] Although we have applied the methods presented here to determine both local amplification and anelastic attenuation for fundamental mode Rayleigh waves from periods of $24 \mathrm{~s}$ to $100 \mathrm{~s}$ across the western U.S., only the results at $30 \mathrm{~s}$ and $60 \mathrm{~s}$ are presented to demonstrate the method. In principle, the same method can also be applied to higher mode and Love waves. The inversion for 3-D velocity, density, and anelastic structures based on estimates of local amplification and attenuation will be the subject of future contributions.

\section{Theoretical Background}

[9] In this section, we derive the basic equations used to study Rayleigh wave amplification and attenuation based on vertical component measurements. While it is not presented here, a similar derivation can be performed for Love waves.

[10] For a smoothly varying earth model, the single frequency single mode 2-D surface wave potential $\chi_{2 D}$ approximately satisfies the 2-D homogenous wave equation [Tanimoto, 1990; Tromp and Dahlen, 1993]

$$
\frac{1}{c(\mathbf{r})^{2}} \frac{\partial^{2} \chi_{2 D}(\mathbf{r}, t)}{\partial t^{2}}=-\frac{2 \alpha(\mathbf{r})}{c(\mathbf{r})} \frac{\partial \chi_{2 D}(\mathbf{r}, t)}{\partial t}+\nabla^{2} \chi_{2 D}(\mathbf{r}, t),
$$

where $c$ and $\alpha$ are the phase velocity and the attenuation coefficient, respectively, and $\mathbf{r}$ is the position on the 2-D surface. $\alpha$ is related to the attenuation quality factor $\mathrm{Q}$ by $\alpha=\pi f / C Q$, where $C$ is the group velocity and $f$ is the wave frequency [e.g., Prieto et al., 2009]. Based on conservation of energy flux, Tromp and Dahlen [1992] showed that the 3-D Rayleigh wave vertical component displacement wavefield observed on the surface, $\boldsymbol{u}_{3 D}$, can be related to the 2-D potential, $\chi_{2 D}$, through a local amplification factor, $\beta$, such that

$$
u_{3 D}(\mathbf{r}, t)=A(\mathbf{r}) e^{i \omega[t-\tau(\mathbf{r})]}=\beta(\mathbf{r}) \chi_{2 D}(\mathbf{r}, t),
$$

where $A$ and $\tau$ are the observed amplitude and phase travel time at frequency $\omega$ of the 3-D wavefield. The local amplification, $\beta$, can be expressed as

$$
\beta(\mathbf{r})=\left(\frac{c(\mathbf{r}) C(\mathbf{r}) I_{1}(\mathbf{r})}{c^{\prime} C^{\prime} I_{1}^{\prime}}\right)^{-\frac{1}{2}},
$$

where $c$ is the phase velocity and $I_{1}$ is the depth integral of density $\rho$ (from the center of the earth, $z=0$, to the surface, $z=a$ ) modulated by the normalized eigenfunctions beneath location $\mathbf{r}$ :

$$
I_{1}=\int_{0}^{a} \rho(z)\left(U(z)^{2}+V(z)^{2}\right) z^{2} d z .
$$

$U$ and $V$ are the normalized eigenfunctions $(U(a)=1)$ for Rayleigh waves in the vertical and horizontal directions, respectively. Note that the $c^{\prime}, C^{\prime}$, and $I_{1}^{\prime}$ in equation (3) represent reference quantities based on an arbitrary 1-D reference model and the amplification $\beta$ defined here is dimensionless. This is different from the absolute amplification $\left(c \mathrm{CI}_{1}\right)^{-1 / 2}$ defined in Tromp and Dahlen [1992]. Because of this, the 2-D potential $\chi_{2 D}$ used here has the same dimensions as displacement. While the absolute amplification factor $\left(c C I_{1}\right)^{-1 / 2}$ may potentially be constrained when the absolute energy flux is known, such information is usually unavailable for regional studies based on teleseismic earthquakes. In this study, we will focus on resolving the spatial variation of the dimensionless amplification $\beta$. 
[11] Substituting the 2-D wave potential from equation (2)

$$
\chi_{2 D}(\mathbf{r}, t)=\frac{A(\mathbf{r})}{\beta(\mathbf{r})} e^{i \omega[t-\tau(\mathbf{r})]}
$$

into equation (1), the real part of the resulting expression is

$$
\frac{1}{c^{2}}=\nabla \tau \cdot \nabla \tau-\frac{\nabla^{2}(A / \beta)}{\omega^{2}(A / \beta)} .
$$

(For the sake of conciseness, we drop the dependency of all variables on $\boldsymbol{r}$ ). Equation (5) is the basic equation used in the Helmholtz tomography method [Lin and Ritzwoller, 2011a], in which we previously assumed a constant amplification term $(\beta=1)$. The gradient of travel time in equation (5) gives the apparent phase slowness and, with the frequency dependence, the Laplacian term is a finite-frequency correction of the apparent phase slowness measurement. For highfrequency applications, the Laplacian term can be dropped and equation (5) becomes the eikonal equation

$$
\frac{\hat{k}}{c}=\nabla \tau,
$$

where $\hat{k}$ describes the direction of wave propagation. Equation (6) is the basic equation used in eikonal tomography [Lin et al., 2009], which describes geometrical ray theory.

[12] In contrast to equation (5), the imaginary part of the resulting expression is

$$
-\frac{2 \alpha}{c}=\frac{2 \nabla(A / \beta) \cdot \nabla \tau}{A / \beta}+\nabla^{2} \tau,
$$

in which the attenuation constant is seen to be related to the 2-D amplitude decay in the direction of wave propagation (first term on the right hand side (RHS) of equation (7)) corrected by the Laplacian of the phase travel time field (second term on the RHS of equation (7)). The Laplacian correction term here basically describes the curvature of the phase front that results from elastic focusing/defocusing. To investigate the local amplification $\beta$, the first term on the RHS of equation (7) is expanded to give the following:

$$
\frac{2 \nabla \beta \cdot \nabla \tau}{\beta}-\frac{2 \alpha}{c}=\frac{2 \nabla A \cdot \nabla \tau}{A}+\nabla^{2} \tau .
$$

The RHS of equation (8) contains only quantities that are directly measurable from the surface wavefield $(A, \tau)$ of a single-frequency vertical component Rayleigh wave on the earth's surface. The unmeasurable quantities that we wish to infer $(c, \beta, \alpha)$ appear exclusively on the left hand side (LHS), although we may assume that phase speed $c$ has been locally estimated based on the real part expression, equation (5) or (6). There are, therefore, two remaining unknowns: local amplification $\beta$ appears only in the first term on the LHS and the attenuation coefficient $\alpha$ only in the second term on the LHS.

[13] We refer here to the first term on the RHS of equation (8) as the "apparent amplitude decay" (in the direction of wave propagation). The combination of both terms on the RHS is referred to as the "corrected amplitude decay," where the Laplacian of the travel time field provides the correction for focusing and defocusing. On the LHS of equation (8), the first term is referred to as the local "amplification term" for the Rayleigh wavefield. There is, therefore, a potential terminological confusion between the local amplification $\beta$, which is a property of the Rayleigh wave, and the amplification term, which is a mathematical construct. The second term on the LHS is the "anelastic attenuation" term.

[14] Thus, from equation (8) it is clear that the corrected amplitude decay will differ from the anelastic attenuation term by the local amplification term. However, the amplification term on the LHS contains the gradient of the phase travel time and will vary with the direction of wave propagation whereas the attenuation term is azimuth independent. In the approach described below, we attempt to separate the effect of attenuation and amplification by examining the directional dependence of the corrected amplitude decay measurements.

\section{Method}

[15] We closely follow the method described by Lin et al. [2009] and Lin and Ritzwoller [2011a] to select the fundamental mode Rayleigh wave data and construct both phase travel time and amplitude maps across USArray Transportable Array. More than 900 earthquakes (Figure 1b) with $M_{s}>$ 5.0 between 1 January 2007 and 30 June 2011 are used. Note that the 2-D wave equations described in the previous section are all derived from local wave propagation, which does not depend on source properties such as radiation pattern and earthquake magnitude. An example of the observed $60 \mathrm{~s}$ Rayleigh wave phase travel time and amplitude maps for a 2009 Kuril Islands earthquake is shown in Figure 2. Unlike phase travel time measurements, which mostly increase monotonically in the direction of wave propagation, the observed amplitude measurements often vary in both radial and transverse directions due to the combination of interference, focusing/defocusing, attenuation, and amplification effects. The striping pattern in Figure $2 b$, for example, is probably due to the interference between waves coming from the continental and oceanic flanks of the great circle path. In this section, we will use $60 \mathrm{~s}$ Rayleigh wave measurements, which are most sensitive to uppermost mantle structure, to demonstrate our method.

\subsection{Corrected Amplitude Decay Measurement}

[16] We now demonstrate the ability to correct for amplitude variations in the direction of wave propagation caused by elastic focusing/defocusing. Figures $3 a$ and $3 b$ compare the apparent amplitude decay $(2 \nabla \mathrm{A} \cdot \nabla \tau / \mathrm{A})$ and the focusing/ defocusing correction $\left(\nabla^{2} \tau\right)$ derived from the amplitude and travel time measurements presented in Figure 2. Our method here differs somewhat from the presentation of Lin and Ritzwoller [2011a], in that here we utilize Gauss' theorem to estimate the Laplacian of phase travel time by performing a contour integral in the gradient of phase travel time. For each location, the contour is defined on the boundary of a $1.2^{\circ} \times 1.2^{\circ}$ box centered at that point. In an attempt to match the resolution between gradient and Laplacian operators, the apparent amplitude decay is also estimated based on the average value within the same box. A clear anticorrelation is observed between the apparent amplitude decay and the 
(a)
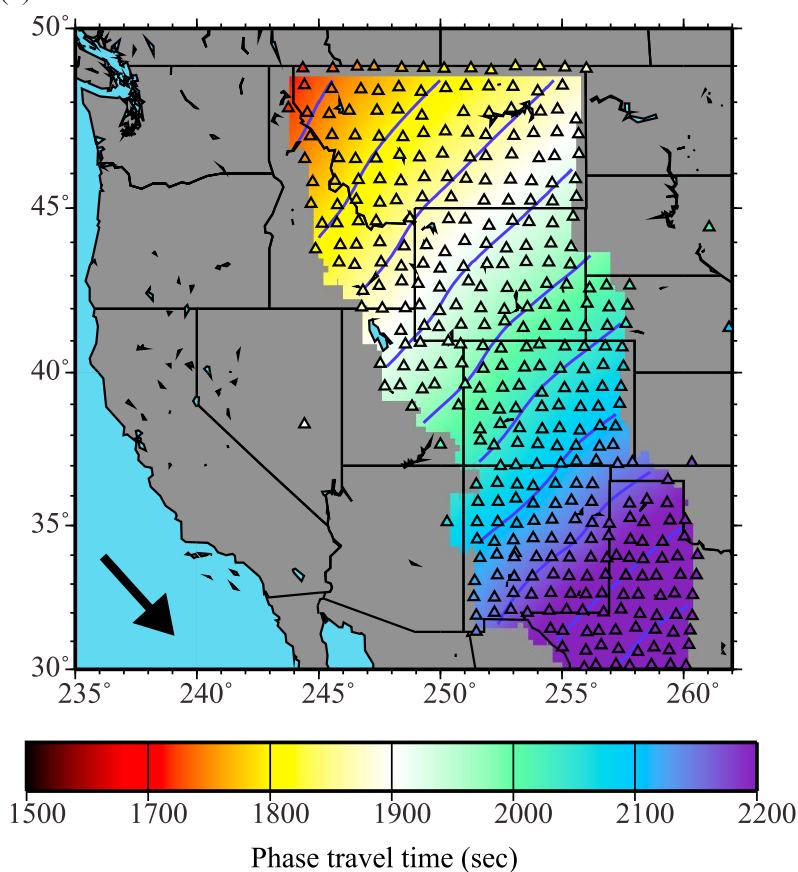

(b)

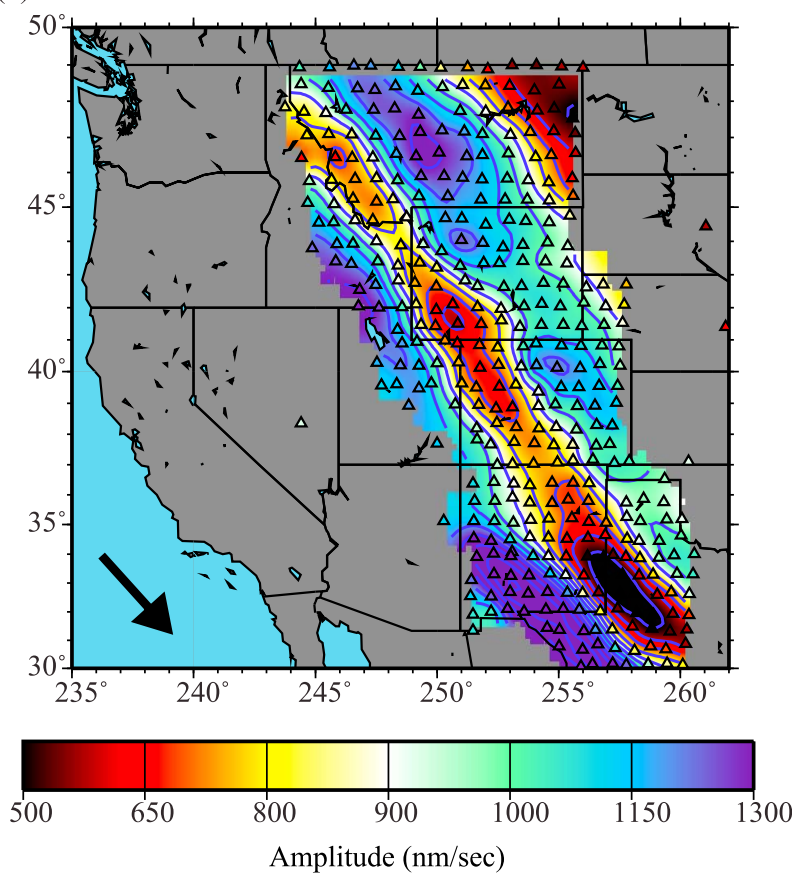

Figure 2. The $60 \mathrm{~s}$ Rayleigh wave observed (a) phase travel time and (b) amplitude maps for the 7 April 2009 earthquake near the Kuril Islands $(M s=6.8)$. The stations where phase travel time and amplitude measurements are obtained are shown as triangles. Contours are separated by intervals of $60 \mathrm{~s}$ in Figure $2 \mathrm{a}$ and $100 \mathrm{~nm} \mathrm{~s}^{-1}$ in Figure $2 \mathrm{~b}$. The arrows indicate the approximate direction of wave propagation.

focusing/defocusing correction shown in Figures 3a-3c, as suggested by equation (8). Note that the same Kuril Island earthquake was also used by Lin and Ritzwoller [2011a] to demonstrate the principle of Helmholtz tomography and was compared with equation (5). By removing the focusing/ defocusing effect, the corrected amplitude decay (Figure $3 \mathrm{~d}$ ) eliminates the apparent striping bias and is better correlated with the boundaries of known velocity structures (Figure 4). For example, strong amplitude decay (red) in western Wyoming is coincident with the boundary between the Yellowstone/Snake River Plain slow anomaly and the southwestern Wyoming fast anomaly. The increase of amplitude (blue) in western Colorado, on the other hand, is coincident with the boundary between the Colorado Plateau/ southwestern Wyoming fast anomalies and the southern Rockies slow anomaly. The fact that the corrected amplitude decay map (Figure 3d) is well correlated with the observed velocity boundaries, however, suggests that the corrected amplitude decay actually is dominantly affected by local amplification rather than anelastic attenuation. The local amplification $\beta$ is expected to correlate with velocity structure as shown in equation (3).

[17] Due to the dependence of the amplification term on the phase travel time gradient (equation (8)), the observed corrected amplitude decay is expected to vary with wave propagation direction. In Figure 5, we examine measurements with waves propagating in two primary directional bands: southeast $\left(120^{\circ}\right.$ to $150^{\circ}$ azimuth; Figures $\left.5 \mathrm{a}-5 \mathrm{c}\right)$ and northwest $\left(300^{\circ}\right.$ to $330^{\circ}$ azimuth; Figures $\left.5 \mathrm{~d}-5 \mathrm{f}\right)$, where the amplification terms are expected to have exactly opposite effects. More than 80 earthquakes are averaged in each of the two azimuthal bands. As in previous studies [Lin et al., 2009;
Lin and Ritzwoller, 2011a], the direction of wave propagation for each earthquake at each location is determined from the gradient of phase travel time (equation (6)). In the overlapping region, the average apparent amplitude decay, focusing/defocusing correction, and corrected amplitude decay for waves coming from the northwest (Figures 5a-5c) are largely consistent with the Kuril island earthquake measurements (Figure 3). Anticorrelation between the apparent amplitude decay and the focusing/defocusing correction is again observed for both directions (Figures $4 \mathrm{a}$ and $4 \mathrm{~b}$ and Figures $4 \mathrm{~d}$ and $4 \mathrm{e}$ ), particularly where the focusing/ defocusing correction is strong. Clear anticorrelation can also be observed for the corrected amplitude decay for the two opposite directions (Figures 5c and 5f), which suggests that not only is equation (8) valid but also that the amplification effect is much stronger than the variation of anelastic attenuation at this period. Note that, in principle, the focusing/ defocusing effect can also be used to determine velocity structure variations. However, this is beyond the scope of this study.

\subsection{Amplification Map}

[18] To determine the amplification effect accurately, the effect of attenuation must be removed from the corrected amplitude decay measurements. We do this by studying the directional dependence of the measurements. With the dot product in the amplification factor (equation (8)), we expect the observed corrected amplitude decay to have a $360^{\circ}$ azimuthal periodicity at each location where the variation should be independent from attenuation. An example of observed directionally dependent corrected amplitude decay measurements on a 9 point grid ( 3 by 3 grid with $0.6^{\circ}$ 
(a)

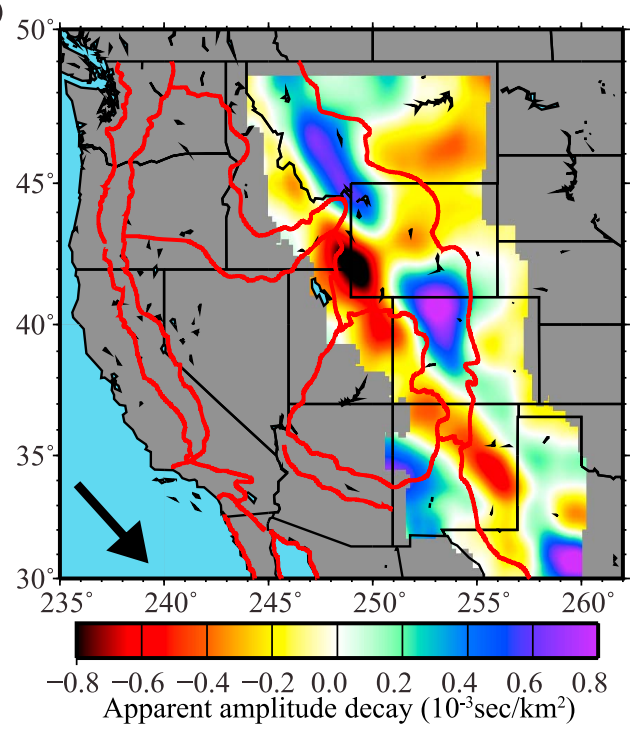

(c)

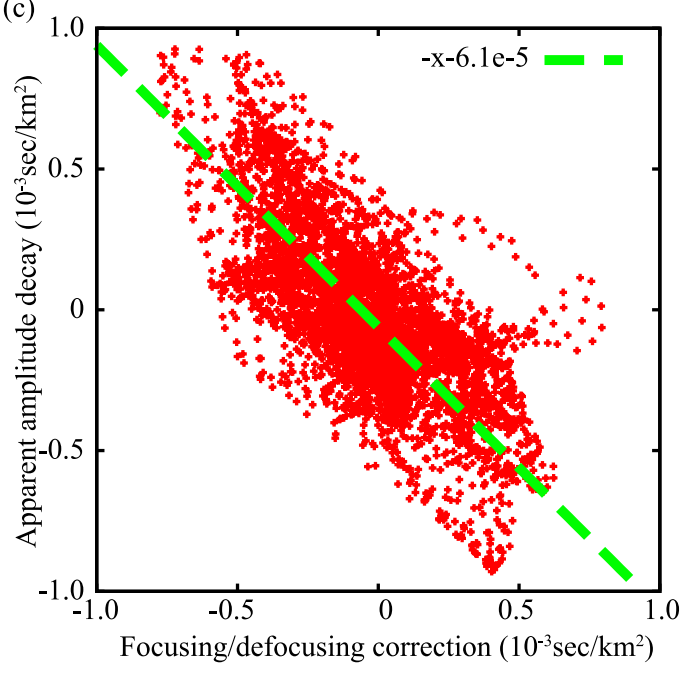

(b)
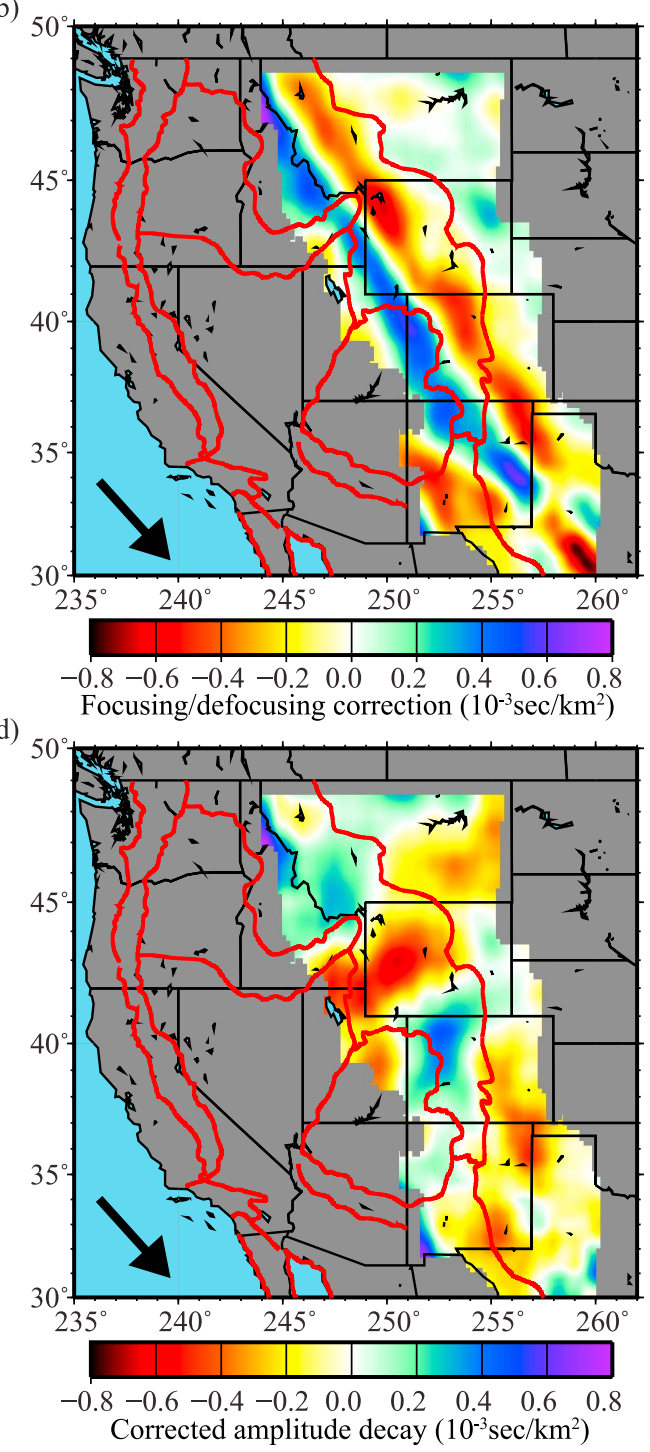

Figure 3. (a) The apparent amplitude decay (first term on the RHS of equation (8)) derived from Figures $2 \mathrm{a}$ and $2 \mathrm{~b}$. (b) The focusing/defocusing correction term (second term on the RHS of equation (8)) derived from Figure 2a. (c) The relation between the apparent amplitude decay and the focusing/defocusing correction term based on Figures $3 \mathrm{a}$ and $3 \mathrm{~b}$ where each point represents the result at a grid point on the maps. The green dashed line is the best fitting straight line with a slope of -1 . The value of the intercept is also shown. (d) The corrected amplitude decay map derived from Figures 3a and $3 b$ based on the RHS of equation (8). The arrows in Figures 3a, 3b, and 3d indicate the approximate direction of wave propagation.

spacing) centered at a point in western Wyoming (star in Figure 1a) is shown in Figure 6a. The scattering of the measurements potentially represents measurement errors due to effects that are not accounted for in our 2-D approach (e.g., due to interference between the fundamental mode Rayleigh wave and other wave types) and only the statistical average over many earthquakes is reliable. Here, similar to previous studies on the directional dependence of phase velocity measurements [Lin et al., 2009, 2011a; Lin and Ritzwoller, 2011a, 2011b], the mean and the standard deviation of the mean for all available measurements within each $20^{\circ}$ azimuthal bin are used to estimate the corrected amplitude decay and its uncertainty for each direction (Figure 6b). Note that the 9 point averaging scheme is applied here to reduce measurement uncertainty [Lin et al., 2009], but it also degrades the resolution to $\sim 200 \mathrm{~km}$. A clear $360^{\circ}$ azimuthal periodicity is observed in Figures $6 \mathrm{a}$ and $6 \mathrm{~b}$, where a large positive amplitude decay (or amplification) is associated with waves propagating toward the northwest (from the southwestern Wyoming fast anomaly into the Yellowstone/Snake River Plain slow anomaly) and a negative amplitude decay is associated with waves propagating in the opposite direction.

[19] Based on the observation of the directionally dependence of such measurements (e.g., Figure 6b), we fit a single sine function with $360^{\circ}$ periodicity to the corrected amplitude decay measurements at each location to estimate both the maximum amplification direction and the amplification 
(a)

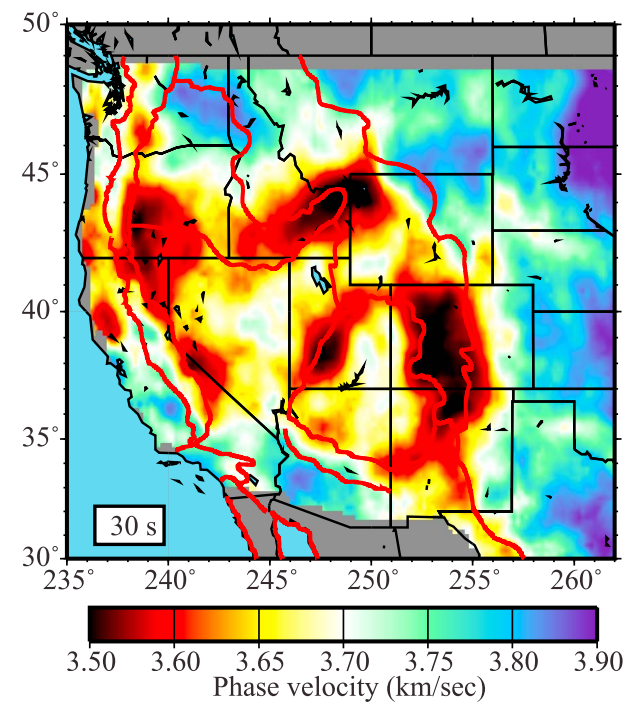

(b)

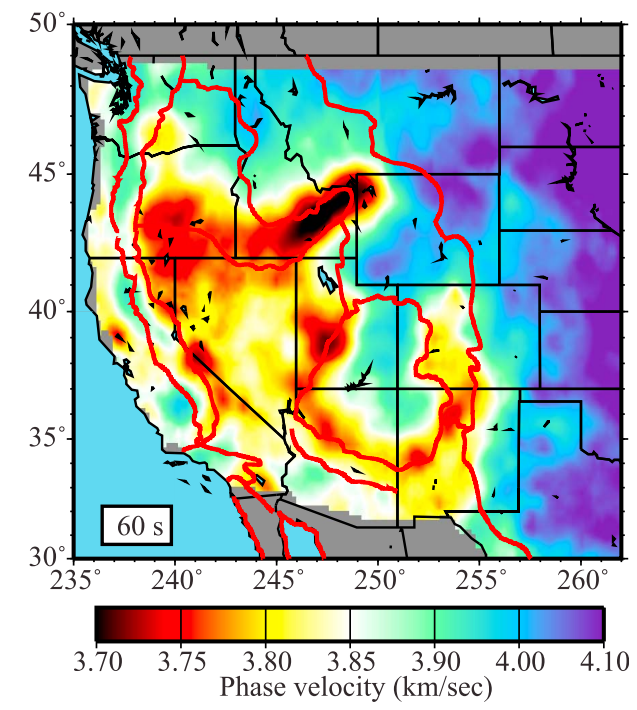

Figure 4. The (a) $30 \mathrm{~s}$ and (b) $60 \mathrm{~s}$ Rayleigh wave phase velocity maps constructed using Helmholtz tomography [Lin and Ritzwoller, 2011a].
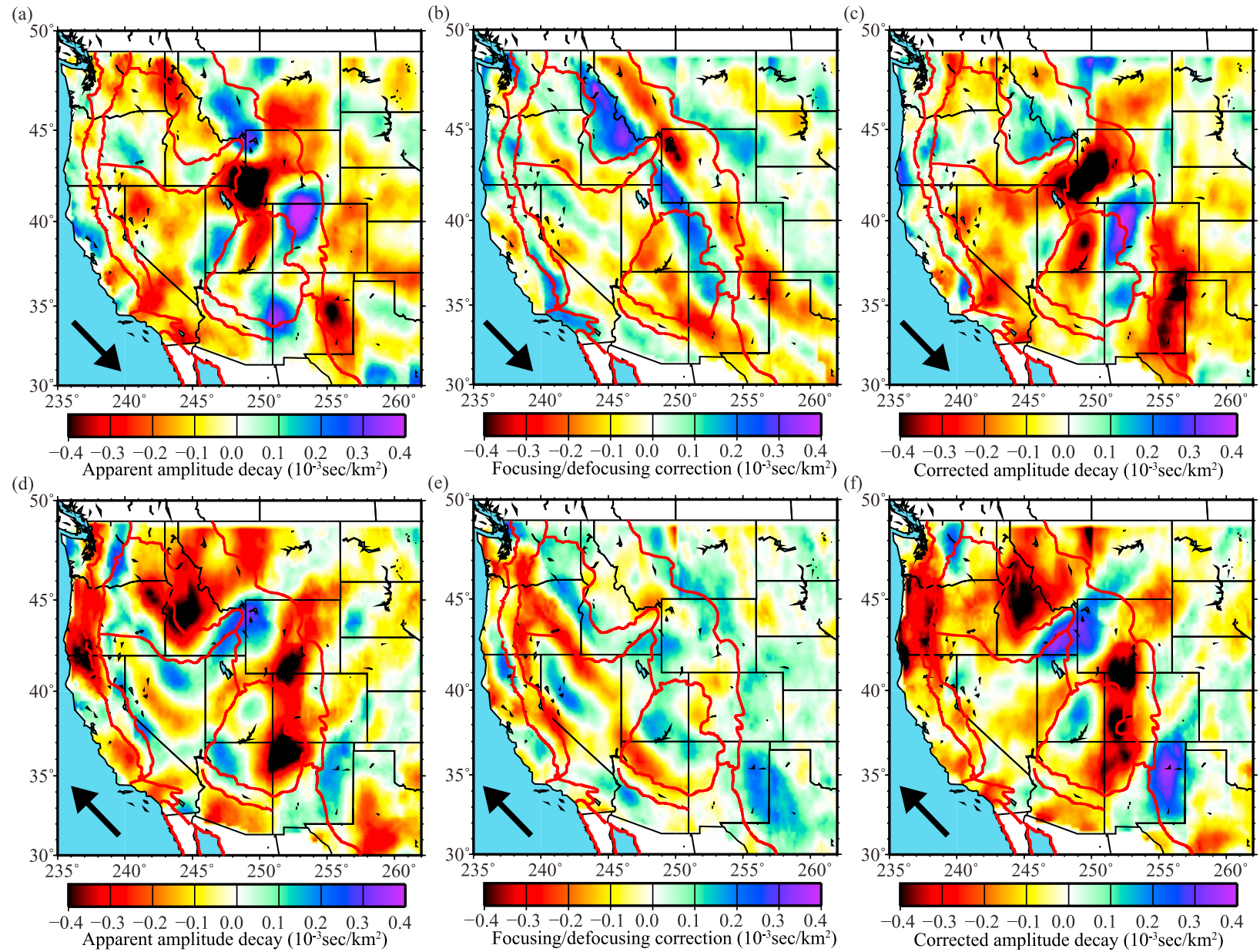

Figure 5. $(\mathrm{a}-\mathrm{c})$ The average apparent amplitude decay, focusing/defocusing correction, and corrected amplitude decay, respectively, for all measurements obtained from waves propagating in the southeast direction $\left(120^{\circ}\right.$ to $150^{\circ}$ azimuth angle). The arrows indicate the approximate direction of wave propagation. (d-f) Same as Figures $5 \mathrm{a}-5 \mathrm{c}$ except for waves propagating in the northwest direction $\left(300^{\circ}\right.$ to $330^{\circ}$ azimuth angle). 


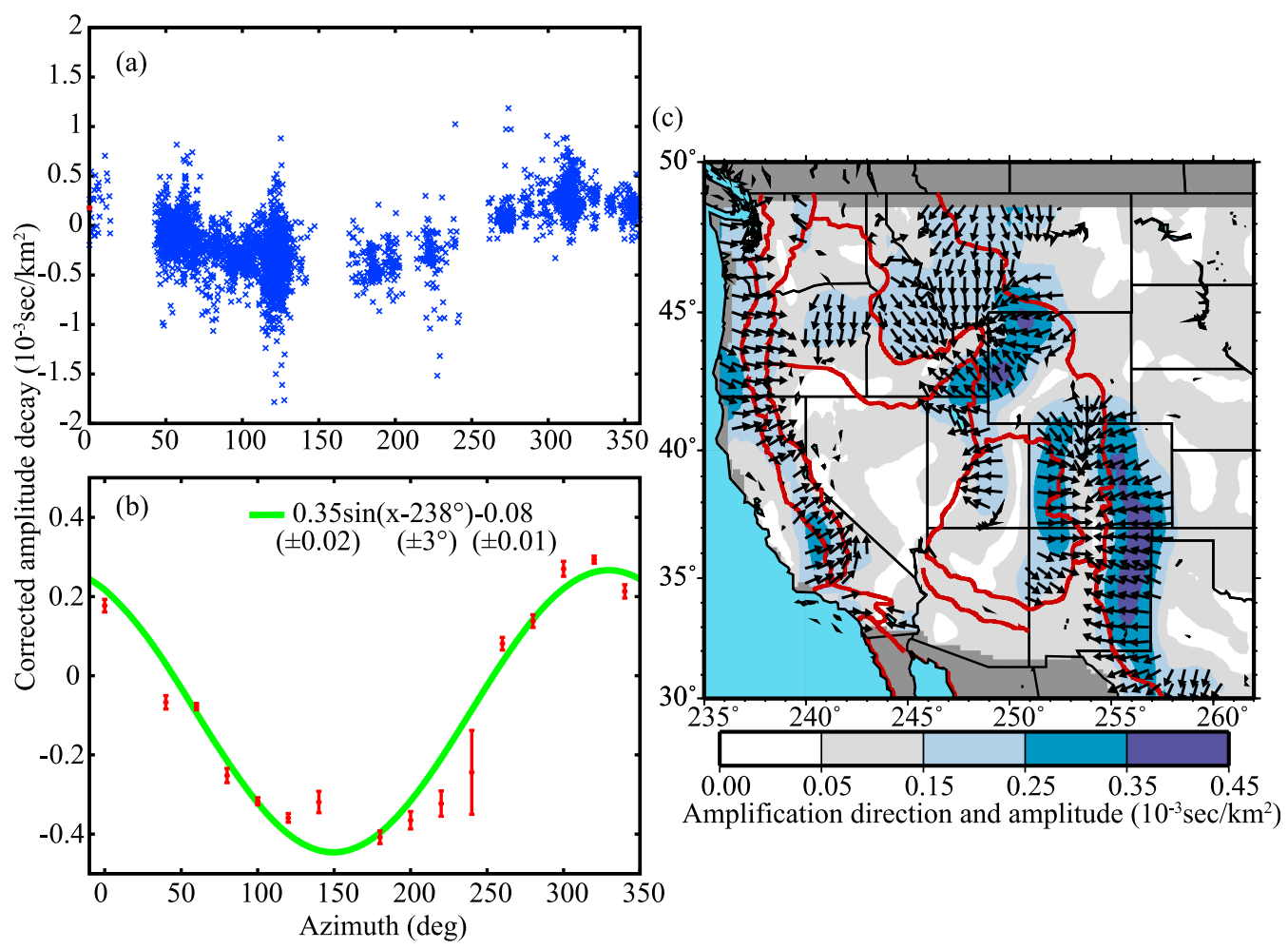

Figure 6. (a) The $60 \mathrm{~s}$ Rayleigh wave directionally dependent corrected amplitude decay measurements near a point in western Wyoming (star in Figure 1a). (b) Statistical summary of measurements shown in Figure 6a. Each error bar is the mean and the standard deviation of the mean of all measurements within each $20^{\circ}$ bin. The solid green line is the best fitting curve of a $360^{\circ}$ periodicity sine function. Both the best fitting parameters and their uncertainties (within the parentheses) are also shown. The maximum amplification direction is $\sim 328^{\circ}$ azimuth and the amplification amplitude is $\sim 0.35 \times 10^{-3} \mathrm{~s} / \mathrm{km}^{2}$. (c) The observed $60 \mathrm{~s}$ Rayleigh wave maximum amplification directions and amplification amplitudes. The arrows present the maximum amplification directions with amplification amplitude larger than $0.1510^{-3} \mathrm{~s} \mathrm{~km}^{-2}$. The amplification amplitude is shown in the background.

amplitude. We associate these two parameters with the 2-D vector $2 \nabla \beta|\nabla \tau| / \beta$ based on equation (8). Because the anelasticity term is not directionally dependent, the mean of the sine function can be used to estimate the attenuation factor $\alpha$. This will be the subject of further discussion in section 5. The observed amplification amplitudes for the $60 \mathrm{~s}$ Rayleigh waves across the whole array are summarized in Figure $6 \mathrm{c}$, where the maximum amplification directions are also plotted where the amplification amplitudes are large $\left(>0.1510^{-3} \mathrm{~s} / \mathrm{km}^{2}\right)$. As expected, large amplification amplitude is observed near sharp velocity structural boundaries (Figure 4) with maximum amplification directions pointing toward the slow anomalies (e.g., Yellowstone/Snake River Plain and Southern Rockies). Note that a very similar pattern is observed for the $1 \psi\left(360^{\circ}\right.$ periodicity) azimuthally anisotropic phase velocity measurements. This apparent bias has been attributed to finite-frequency sensitivity to backscattering [Lin and Ritzwoller, 2011b].

[20] By approximating the phase slowness $1 / \mathrm{c}$ as the absolute value of the apparent slowness $|\nabla \tau|$ and using the phase velocity map derived from Helmholtz tomography, we now have an estimate of the normalized amplification gradient vector field $\nabla \beta / \beta$. Because this vector field is essentially derived from the amplification differences observed between nearby stations, we invert the amplification $\beta_{\mathrm{i}}$ at each station location based on this vector field by minimizing the misfit functional:

misfit $=\sum_{i, j}\left[\frac{2\left(\beta_{j}-\beta_{i}\right)}{\left(\beta_{j}+\beta_{i}\right)}-\left.\frac{\nabla \beta}{\beta}\right|_{\frac{\left(\mathbf{r}_{i}+\mathbf{r}_{j}\right)}{2}} \cdot\left(\mathbf{r}_{j}-\mathbf{r}_{i}\right)\right]^{2} \forall\left|\mathbf{r}_{j}-\mathbf{r}_{i}\right|<120 \mathrm{~km}$,

where $i, j$ are the station indices, $\mathbf{r}$ is the station location, and $\left(\mathbf{r}_{\mathrm{i}}+\mathbf{r}_{\mathrm{j}}\right) / 2$ is the midpoint between station pair $(i, j)$ where the normalized amplification gradient vector is evaluated. In order only to evaluate the closest station pairs that contribute to the observed amplification gradient vector field, the summation is taken over all station pairs that are within $120 \mathrm{~km}$ of one another. Because any set of amplification measurements, $\beta_{\mathrm{i}}$, scaled by an arbitrarily constant will have the same misfit, in order to obtain a unique solution we set the average amplification of all stations to unity by using the freedom to select an arbitrarily reference model in equation (3). Also, to linearize the inversion we assume that the variation of amplification is small such that $\left(\beta_{\mathrm{i}}+\beta_{\mathrm{j}}\right) / 2$ is approximately equal to 1 . The inverted amplification map based on the maximum amplification directions and amplification amplitudes shown in Figure $6 \mathrm{~b}$ and equation (9) is shown in Figure $7 \mathrm{a}$, where we interpolate the result to cover the whole 
(a)

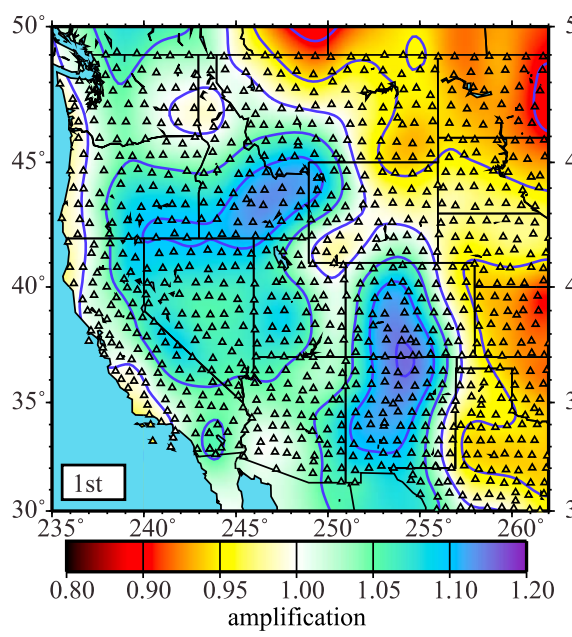

(b)

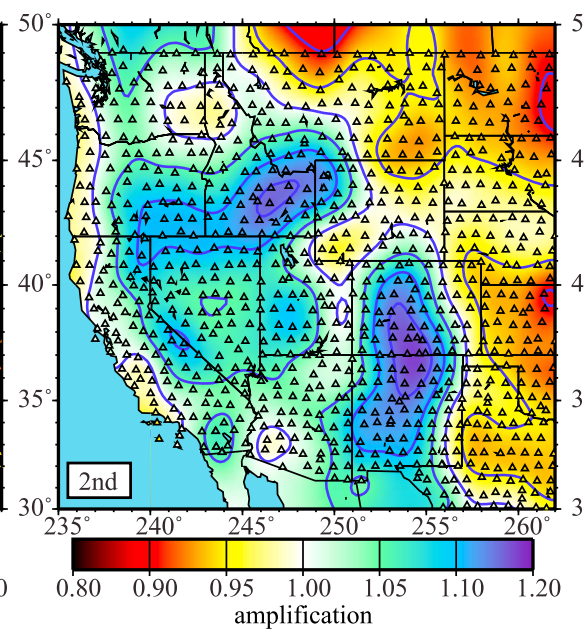

(c)

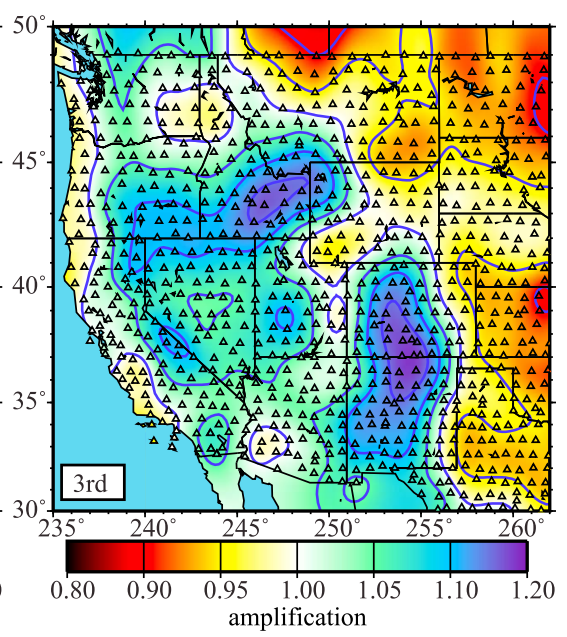

Figure 7. (a) The $60 \mathrm{~s}$ Rayleigh wave amplification maps derived (equation (9)) from the maximum amplification directions and amplification amplitudes shown in Figure 6c. The triangles show the station locations used in the inversion. (b, c) Same as Figure 7a except after the second and third iterations, respectively.

area based on the minimum curvature surface-fitting method [Smith and Wessel, 1990]. The estimated amplification map is intrinsically smoothed considering the $\sim 200 \mathrm{~km}$ resolution of our corrected amplitude decay measurements.

[21] The amplification map for the $60 \mathrm{~s}$ Rayleigh wave (Figure 7a) is consistent with expectations based on equation (3) where high and low amplifications are associated with slow and fast phase speed anomalies (Figure 4), respectively. For example, in regions like the Southern Rockies and the Yellowstone/Snake River Plain slow anomalies, slowly propagating waves are associated with large amplifications in order to satisfy the conservation of energy flux. Potential bias in the inversion of amplification can be reduced iteratively. In each iteration, we use the inverted station amplification $\beta_{\mathrm{i}}^{\text {old }}$ to remove the amplification effect from the raw amplitude measurement (i.e., $\mathrm{A}_{\mathrm{i}}^{\prime}=$ $\left.\mathrm{A}_{\mathrm{i}} / \beta_{\mathrm{i}}^{\text {old }}\right)$ and reevaluate the remnant amplification $\left(\beta_{\mathrm{i}}^{\prime}\right)$ based on the updated corrected amplitude decay measurements $\left(2 \nabla \mathrm{A}^{\prime} \cdot \nabla \tau / \mathrm{A}^{\prime}+\nabla^{2} \tau\right)$ using equation (9) (with $\beta$ now replaced by $\beta^{\prime}$ ). Because the remnant amplification at each station is now closer to 1 , the error in the inversion is reduced. The remnant amplification is then used to correct for the original amplification $\left(\beta_{\mathrm{i}}^{\text {new }}=\beta_{\mathrm{i}}^{\text {old }} \beta_{\mathrm{i}}^{\prime}\right)$. Figures $7 \mathrm{~b}$ and $7 \mathrm{c}$ show the amplification maps after the second and third iteration, respectively. In general, upon each iteration the smallscale amplification anomalies become more pronounced, but the result converges quickly. Considering $\beta=1$ as the 0th iteration, the average amplification difference across the entire study region for the first three iterations are 0.052 , 0.005 , and 0.002 , respectively, with the maximum difference between the second and third iterations being smaller than 0.01 . Hereafter, we use the amplification map produced after three iterations as the final result.

[22] A synthetic test based on 2-D simulations has been performed to validate the method described here and the result is presented in Appendix A. While 3-D wave phenomena, such as mode coupling, are not considered in the 2-D simulations, the synthetic test clearly demonstrates the ability of using our method to account for 2-D wave phenomena, such as focusing/defocusing, and to accurately reconstruct the theoretical local amplification.

\section{Observed Amplification Compared With Amplification Computed From 3-D Models}

[23] In the previous section, we described a method to construct amplification maps for surface waves empirically based on maps of surface wave phase travel time and amplitude. In this section, we validate these maps and demonstrate the potential of incorporating the amplification information into 3-D inversions for shear velocity and density. We perform this validation by comparing the observed $30 \mathrm{~s}$ and $60 \mathrm{~s}$ Rayleigh wave amplification with predictions based on two 3-D shear velocity models in the western U.S.. The two models have both been constructed based on Rayleigh wave phase velocity measurements and are only different in how the density model is prescribed. The $30 \mathrm{~s}$ and $60 \mathrm{~s}$ Rayleigh waves are most sensitive to the lower crust/ uppermost mantle and the upper mantle, respectively.

[24] We use a method similar to that described by Yang et al. [2008], who inverted for a 3-D shear velocity model based on Rayleigh wave phase velocity maps observed between periods of 8 and $10 \mathrm{~s}$. The phase velocity maps used here are constructed based on eikonal and Helmholtz tomography for ambient noise ( 8 to $24 \mathrm{~s}$ ) and earthquake ( 24 to 100 s) data sets, respectively [Lin et al. 2008, 2009; Lin and Ritzwoller, 2011a]. Note that the estimated amplification map allows for a reevaluation of the earlier result of Helmholtz tomography based on equation (5). Only the small-scale anomalies are slightly different from the previous application [Lin and Ritzwoller, 2011a], where a constant amplification term $\beta$ is assumed. At each point, the 1-D shear velocity profile from the surface to $200 \mathrm{~km}$ depth is described by six quadratic $\mathrm{B}$ splines, three in the crust and three in the upper mantle. A velocity discontinuity is allowed between the crust and upper mantle where we fix the Moho depth based on previous receiver function studies [Gilbert, 2012]. We set the $\mathrm{Vp} / \mathrm{Vs}$ ratio to 1.78 in both the crust and the upper 

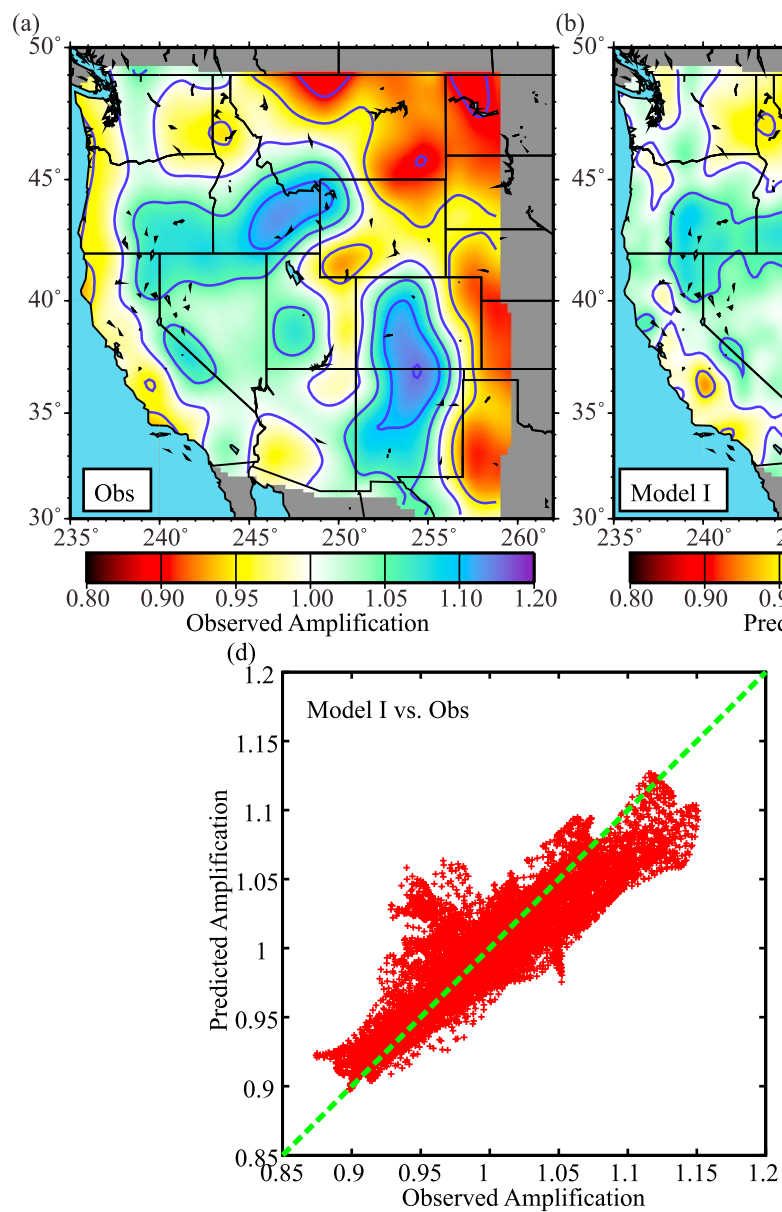
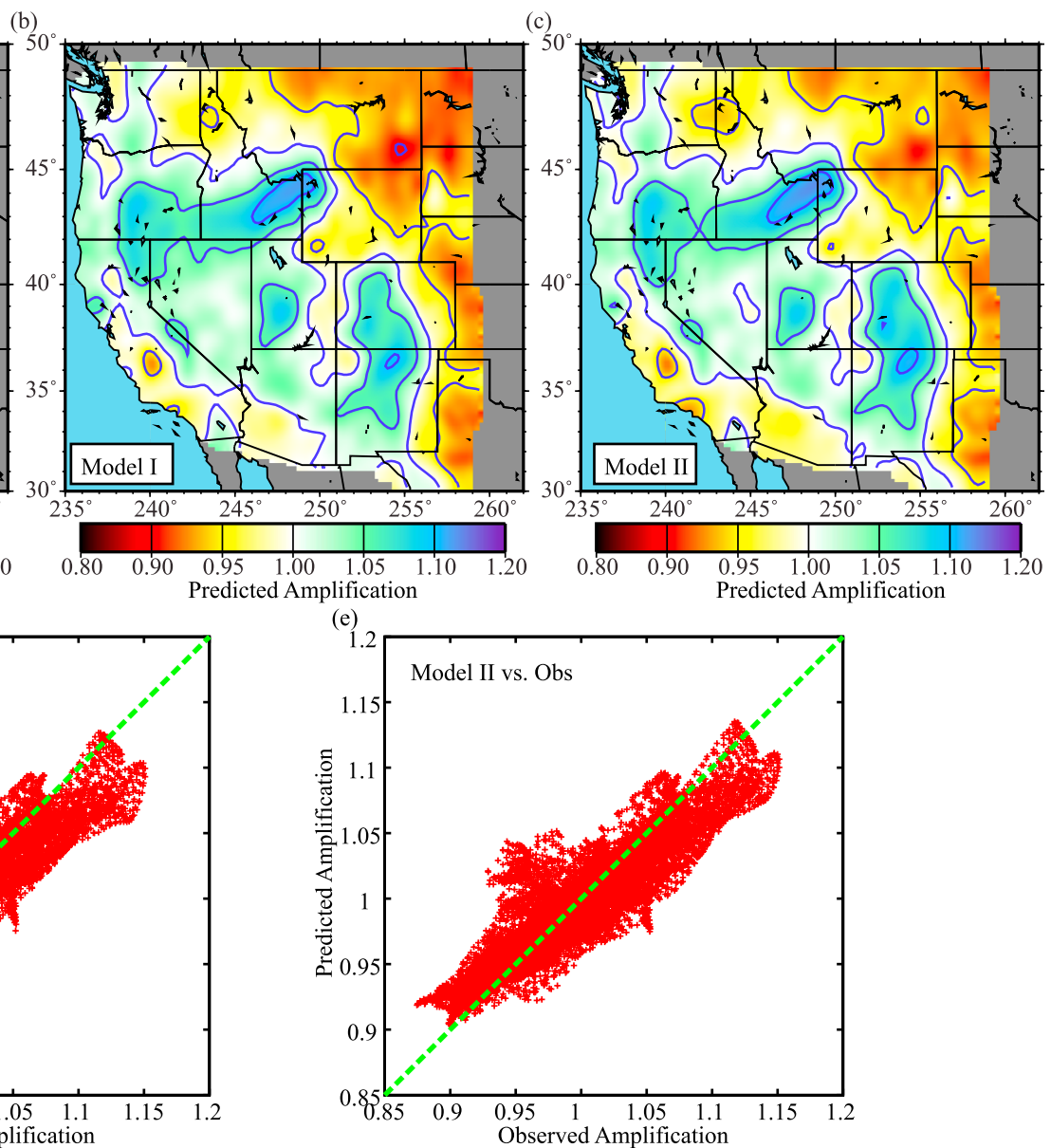

Figure 8. (a) Same as Figure 7c except that only the area with reliable constraints on Moho depth [Gilbert, 2012] is shown. The amplification is normalized by the mean of the amplification in the area and hence is slightly different than Figure 7c. (b) The predicted $60 \mathrm{~s}$ Rayleigh wave amplification map based on the 3-D velocity model with a vertically and laterally homogeneous density structure (Model I). (c) Same as Figure $8 \mathrm{~b}$ but for the laterally homogeneous but vertically inhomogeneous density model (Model II) where a $\sim 20 \%$ density jump is set between the crust and mantle across the Moho. (d) The relation between the predicted and observed amplification shown in Figures $8 \mathrm{a}$ and $8 \mathrm{~b}$ for density Model I where each point represents the results at a grid point on the maps. The green dashed line is the reference line for zero misfit.

(e) Same as Figure 8d but for the relation between Figures $8 \mathrm{a}$ and $8 \mathrm{c}$ for density Model II.

mantle. In the first model (Model I), we assume a nonrealistic homogeneous density model where the crust and mantle have the same density everywhere $\left(3 \mathrm{~g} / \mathrm{cm}^{3}\right)$. In the second model (Model II), we assume a more realistic density model where crust and mantle densities are uniform but differ by $\sim 20 \%$ across the Moho (crust: $2.7 \mathrm{~g} / \mathrm{cm}^{3}$; mantle: $3.38 \mathrm{~g} / \mathrm{cm}^{3}$ ). Both models fit the observed phase velocity dispersion curves acceptably (within errors) and the shear velocity structure, in general, is very similar to that presented by Moschetti et al. [2010b] and Lin et al. [2011a].

\subsection{Rayleigh Waves (60 s)}

[25] For each location, with the 1-D velocity and density models defined in the previous paragraph, all terms in equation (3) can be calculated and the amplification can be predicted. Figure 8 shows a comparison between observed and predicted amplification maps for the $60 \mathrm{~s}$ Rayleigh wave. Note that the area of our 3-D inversion is confined to the region with reliable constraints on Moho depth [Gilbert,
2012] and the amplification maps are all normalized to the mean amplification in the area. The observed amplification maps shown in Figure 8a, therefore, are slightly different from Figure $7 \mathrm{c}$ due to the different area covered.

[26] The pattern of observed amplification (Figure 8a) generally agrees well with the predicted amplification (Figures $8 \mathrm{~b}-8 \mathrm{e}$ ). This validates both the observed amplification map and the assumption that it can be predicted from equation (3). The differences between the predicted maps based on the two different density models are generally small. This is probably due to the insensitivity of $60 \mathrm{~s}$ Rayleigh waves to density variations near the Moho. The sensitivity peaks around $90 \mathrm{~km}$ depth for $60 \mathrm{~s}$ Rayleigh wave phase velocity (see section 5.2), compared to a Moho depth that is on average shallower than $40 \mathrm{~km}$ across our study area [Gilbert, 2012].

[27] Despite the general agreement between the observed and predicted amplification, detailed discrepancies are apparent. First, the variation of amplification is generally 
(a)

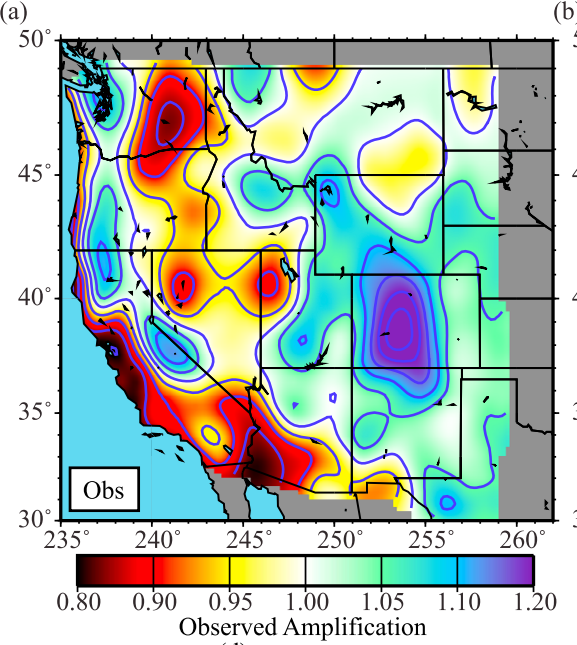

(d)

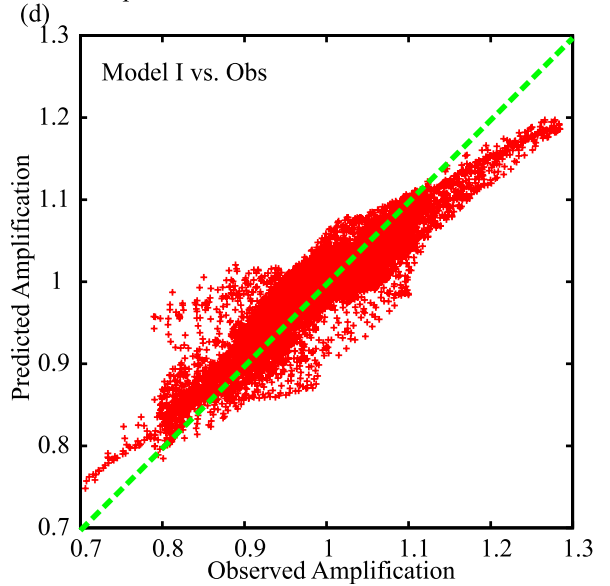

(b)

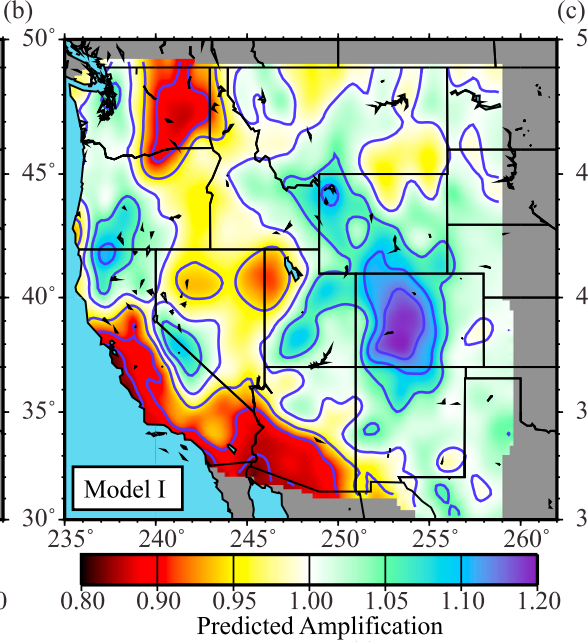

(c)

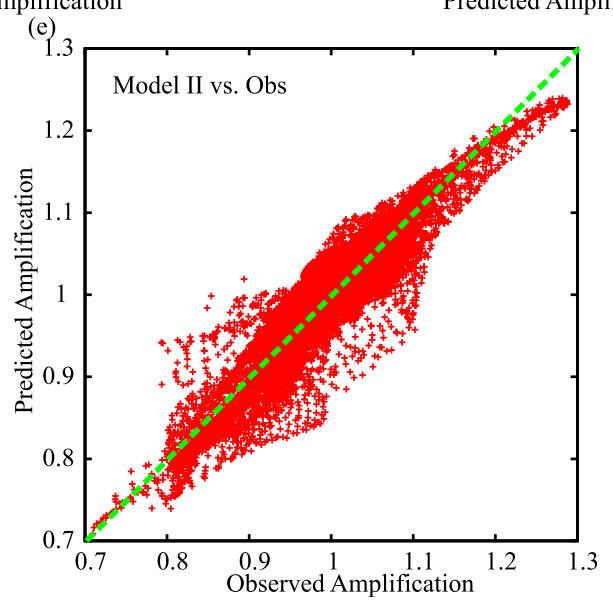

Figure 9. Same as Figure 8 except for the $30 \mathrm{~s}$ Rayleigh wave.

underpredicted by the 3-D models. Aside from small-scale features, which can simply be due to inconsistent resolution between the models and the amplification map, a long wavelength discrepancy is observed where stronger than predicted amplification appears in the tectonically active western U.S. and weaker than predicted amplification appears in the tectonic more stable eastern U.S. While there are several possibilities that will be the subject of a future study, false assumptions about the $\mathrm{Vp} / \mathrm{Vs}$ ratio and density structure are the probable cause of this observed bias. For example, allowing for a more buoyant upper mantle in the western U.S. will produce a slightly stronger amplification in the west and reduce this long wavelength discrepancy. This would be consistent with the assumption of a hot and buoyant upper mantle in the tectonically active western U.S. [e.g., Lowry et al., 2000]. Second, we observe a distinguishable cluster of points in the prediction versus observation scatterplots (Figures 8d and 8e) with weak observed amplification $(<1)$ and strong predicted amplification $(>1)$. Intriguingly, these points mostly correspond to the area near the northwestern Pacific coast where the Juan de Fuca Plate is currently subducting beneath the North American Plate. While uncertainties are expected to be higher near to the edge of the station coverage, allowing for denser mantle within the slab would suppress the local amplification and again reduce the discrepancy.
[28] It must be acknowledged that while density structure can certainly affect the amplification, as suggested by equation (3), variations in velocity structure are still likely to have a dominating effect. Considering that velocity and density structures are frequently correlated, it will prove difficult to recover the density effect without an accurate 3-D velocity model.

\subsection{Rayleigh Waves (30 s)}

[29] Rayleigh waves of $30 \mathrm{~s}$ period, in which the peak phase sensitivity lies near $45 \mathrm{~km}$ depth (see section 6), are expected to be more sensitive to structural variations near the Moho than the $60 \mathrm{~s}$ period waves. Figure 9 shows the comparison between the observed and predicted amplification maps for $30 \mathrm{~s}$ Rayleigh waves in the western U.S. Again, we see a broad agreement between the observed amplification and the predictions based on the 3-D models based on the two different density structures. The fact that even the prediction based on a nonrealistic density model agrees fairly well with the observation suggests that the observed amplification is dominantly controlled by velocity structure.

[30] A detailed comparison, however, shows that the variation in amplification is somewhat underpredicted for the homogeneous density model (Figures $9 \mathrm{~b}$ and $9 \mathrm{~d}$ ). Regions with strong amplification $(>1.1)$, such as the southern Rockies, present a stronger than predicted amplification and 
(a)

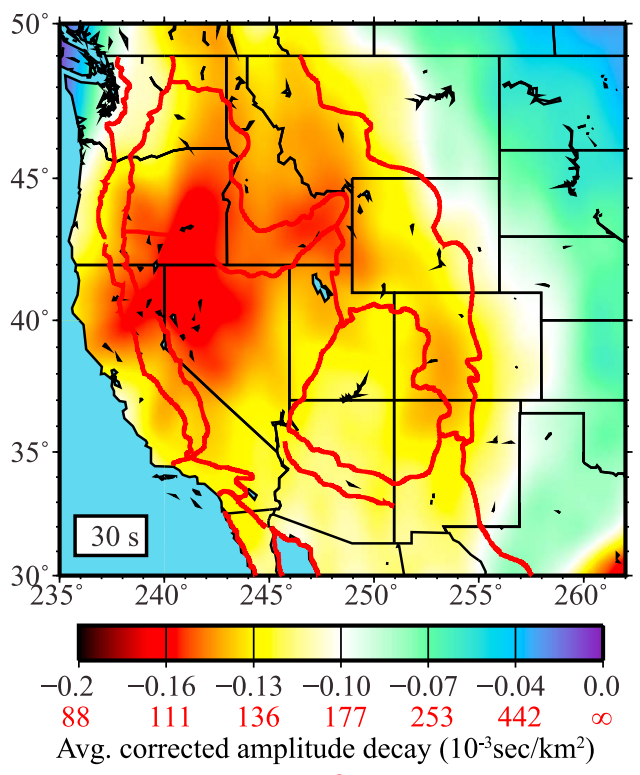

Q (b)

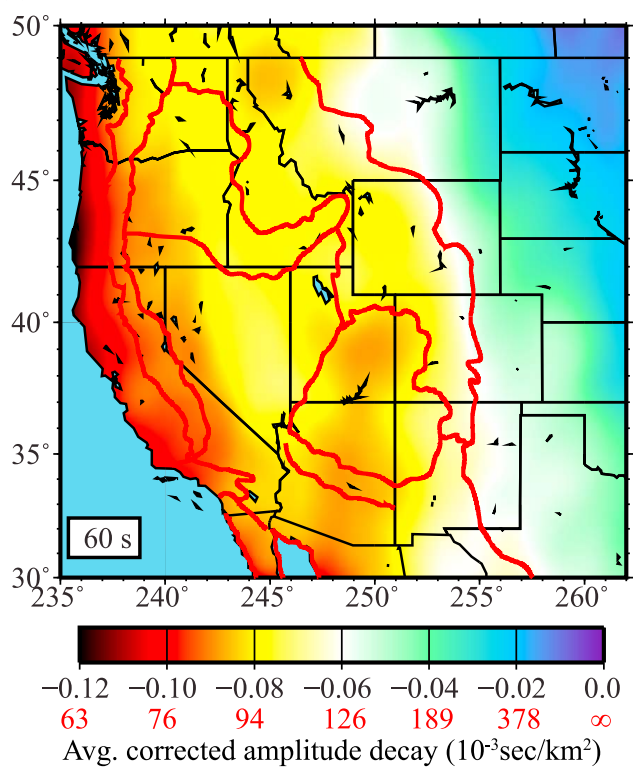

Q

Figure 10. The smoothed (average over a $4^{\circ}$ radius circle) azimuthally weighted corrected amplitude decay for the (a) $30 \mathrm{~s}$ and (b) $60 \mathrm{~s}$ Rayleigh waves. The corresponding approximate $\mathrm{Q}$ values are also shown in red beneath the color bar.

regions with weak amplification $(<0.9)$, such as the Columbia Basin and the Salton Trough, present a weaker than predicted amplification. These strong and weak amplification regions generally correspond to regions with high and low topography, respectively. They also generally correspond to regions with thick and thin crust, respectively, as observed by receiver function studies [e.g., Gilbert, 2012]. In either case, it is reasonable to hypothesize that the stronger and weaker than predicted amplifications are, at least in part, due to differences that are not accounted for in our homogeneous density model.

[31] Further information about the role of density in predicting amplification comes from the second density model that has a more realistic $\sim 20 \%$ density jump across the Moho. The agreement between the observed and predicted amplification, in general, is better than with the vertically homogeneous density model (Figures 9c and 9e). The southern Rockies, for example, still have a slightly underpredicted amplification, but the discrepancy is smaller. Although the Salton Trough and adjacent regions appear to be overcorrected, we do see that most weak amplification regions $(<0.9)$ have better agreement between the predictions and observations. Note that a large discrepancy is observed near the coast in the Pacific northwest. However, both the velocity model and amplification map may not be accurate near the edge of the station coverage. While more tests remain to be completed, we consider these comparisons to be an encouraging first step toward potential velocity and density tomography involving surface wave amplification.

\section{Attenuation Map}

[32] To this point we have concentrated on the observation of local amplification, but attenuation structure can also be estimated based on this method. From equation (8), we may also estimate the attenuation term $-2 \alpha / \mathrm{c}$, but it will be necessary to correct for the amplification term. One method would be to compute the amplification term by using the amplitude map discussed above. However, we can also exploit the fact that the amplification term is azimuthally variable whereas the anelastic attenuation term is not. Thus, by averaging the corrected amplitude decay measurements over azimuth, we can effectively cancel the amplification effect. To avoid bias due to an uneven source distribution, the weight for each measurement is equated with the reciprocal of the number of measurements within each $25^{\circ}$ azimuthal bin. By averaging the measurements at a particular location over azimuth, the first term on the LHS of equation (8) will approximately cancel and the corrected amplitude decay (RHS of equation (8)) will approximate the anelastic attenuation term $(-2 \alpha / \mathrm{c})$. Alternately, attenuation constants could be estimated from the fit of the $360^{\circ}$ periodicity sine function described in section 3.2, but the results are very similar to those presented here.

[33] Because the effect of anelastic attenuation in our amplitude decay measurements is relatively small (e.g., Figure 6b), high-resolution attenuation maps may be more easily biased by details of wave propagation caused by elastic inhomogeneities. Here, we examine large-scale attenuation structure, which should be more accurate and reliable. For each location, we first determine the weighted azimuthal average of corrected amplitude decay. The average value over a $4^{\circ}$ radius circle is used to estimate large-scale attenuation structure $(-2 \alpha / \mathrm{c})$, and the results at periods of $30 \mathrm{~s}$ and $60 \mathrm{~s}$ are summarized in Figure 10. The attenuation quality factor Q may be roughly estimated by approximating the phase and group velocities for $30 \mathrm{~s}$ Rayleigh waves as $3.7 \mathrm{~km} / \mathrm{s}$ and $3.2 \mathrm{~km} / \mathrm{s}$, respectively, and $3.85 \mathrm{~km} / \mathrm{s}$ and $3.6 \mathrm{~km} / \mathrm{s}$ for $60 \mathrm{~s}$ Rayleigh waves (Figure 10). At $30 \mathrm{~s}$ period, Rayleigh waves are most sensitive to uppermost mantle 
(a)

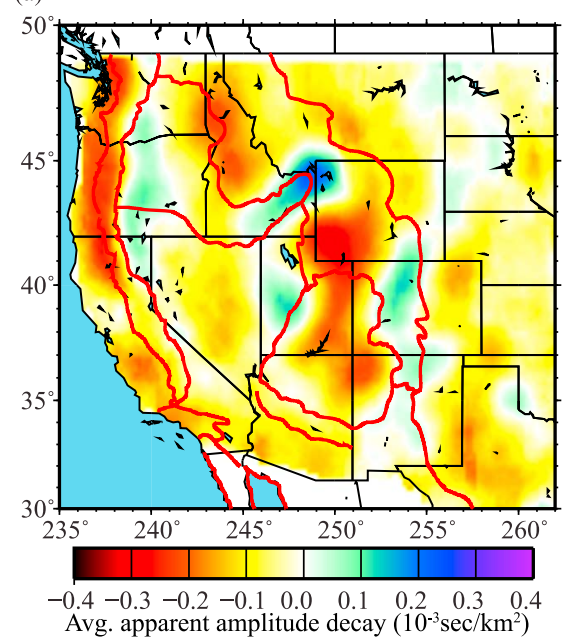

(b)

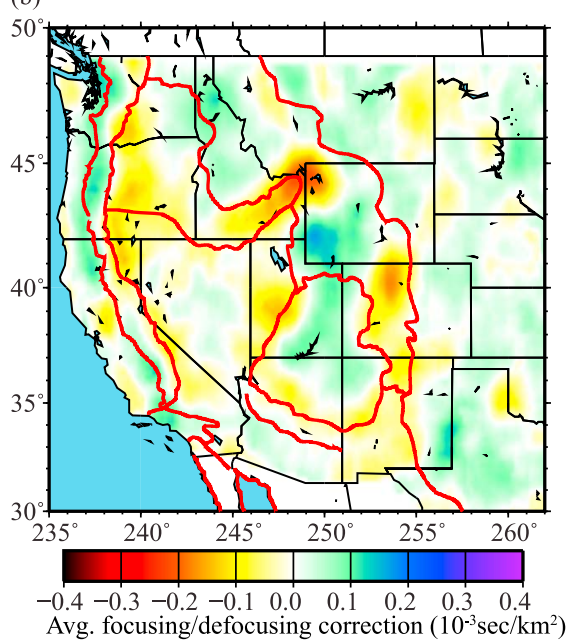

(c)

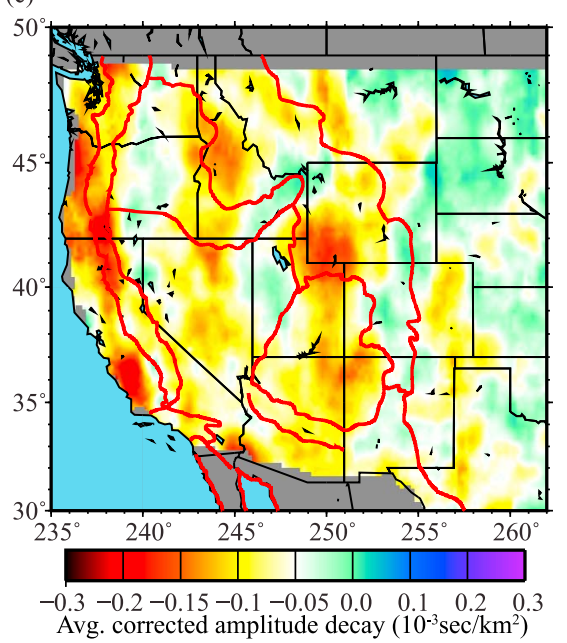

Figure 11. The azimuthally weighted (a) apparent amplitude decay, (b) focusing/defocusing correction, and (c) corrected amplitude decay for all $60 \mathrm{~s}$ Rayleigh wave earthquake measurements.

structure. Clear structural differences are observed between the tectonically active western U.S. and the stable cratonic eastern U.S. where the presumable hotter crust and uppermost mantle in the west is characterized by a higher attenuation and lower Q. More specifically, the largest values of attenuation are observed beneath the High Lava Plains, the Snake River Plain/Yellowstone hot spot track, and the Southern Rockies where significant low shear velocities are also observed (Figure 4) [Lin et al., 2011a]. Note that the anomalous low and high attenuations near the northwestern and southeastern corners, respectively, are near the edge of the station coverage and are probably less reliable.

[34] The $60 \mathrm{~s}$ results for Rayleigh waves are most sensitive to structure between 50 and $150 \mathrm{~km}$ depth and are shown in Figure 10b. Clear structural differences between the tectonically active western U.S. and the more stable cratonic eastern U.S. are again apparent. While $60 \mathrm{~s}$ Rayleigh waves are sensitive to the asthenosphere in the western U.S., they are only sensitive to the lithospheric layer in the east. This result also generally agrees with previous, lower resolution $(>1000 \mathrm{~km})$, global studies [e.g., Dalton and Ekström, 2006b]. Perhaps more surprising is the observation of the largest attenuations between the Colorado Plateau and areas near the Pacific coast where fast anomalies are observed in the phase velocity map (Figure $4 b$ ). In these areas, the fast anomalies are surrounding by slow anomalies where energy loss due to elastic scattering may be important.

[35] To understand the potential biases introduced by complex wave phenomena, the high-resolution (without $4^{\circ}$ averaging) weighted average over azimuth of the apparent amplitude decay (first term on the RHS of equation (8)), the focusing/defocusing correction (second term on the RHS of equation (8)), and corrected amplitude decay measurements (entire RHS of equation (8)) for the $60 \mathrm{~s}$ Rayleigh wave are presented in Figure 11. The azimuthally averaged effect of focusing/defocusing (Figure 11b) is strongly correlated with the phase velocity structure (Figure 4) and with the azimuthally averaged apparent amplitude decay (Figure 11a). The apparent amplitude decay, therefore, is clearly biased by wavefield effects that result from nearby elastic inhomogeneities and should not be used directly to evaluate the attenuation, even when measurements from all azimuths are available. In fact, the focusing/defocusing correction term is of the same order of magnitude as the apparent decay term and the corrected amplitude decay results from a relatively fine cancellation between these two observables. Thus, it is perhaps not surprising that the corrected amplitude decay map (Figure 11c), while mostly showing negative values consistent with the expectation of a positive attenuation constant $\alpha$, does not display strong geological coherence. For example, the Yellowstone/Snake River Plain low-velocity anomaly probably reflects warm mantle temperatures and is expected to be strongly attenuating. Also, the Colorado Plateau high-velocity anomaly is probably cold and weakly attenuating in the mantle. Thus, at present this method appears to have trouble resolving high-resolution attenuation structure. One reason for this may be because the amplitude of the focusing/defocusing correction is relatively poorly constrained at smaller scales and the fine cancellation between the apparent amplitude decay and the correction term is insufficiently accurate to recover high-resolution information about attenuation. The fact that large-scale attenuation at $60 \mathrm{~s}$ period (Figure 10b) appears to retain some of the biases observed for the high-resolution result (Figure 11c), but does not at $30 \mathrm{~s}$, suggests that the biases are probably due to finite-frequency effects, which are stronger at longer periods.

[36] In addition to difficulties in estimating the amplitude of the focusing/defocusing correction term at small scales, we believe that more subtle 3-D wave phenomena may exist that are not accounted for within the 2-D framework of our analysis and may further worsen our estimates of attenuation. If we compare the corrected amplitude decay measurements observed in Figures $5 \mathrm{c}$ and $5 \mathrm{f}$ in detail, we find that while the patterns are generally anticorrelated for waves propagating in opposite directions, the locations of some of the prominent features do not match perfectly in the two maps. For example, near the boundary of the Snake River Plain low-velocity anomaly and the southwestern Wyoming high-velocity anomaly, the significant amplitude decay for waves heading 
(a)

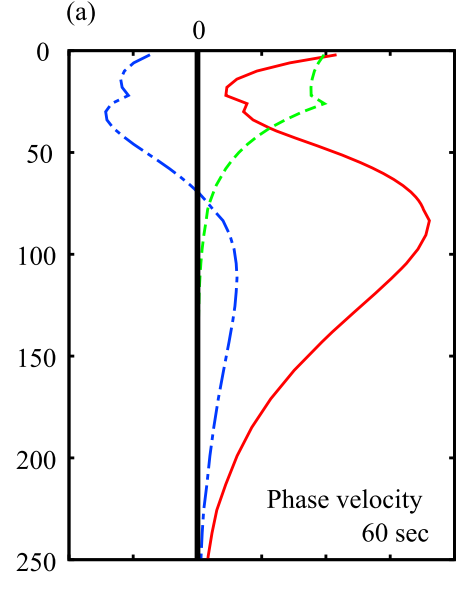

Vs

(e)

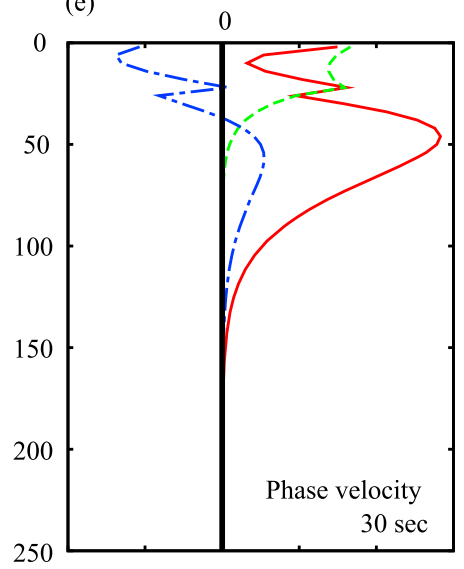

(b)

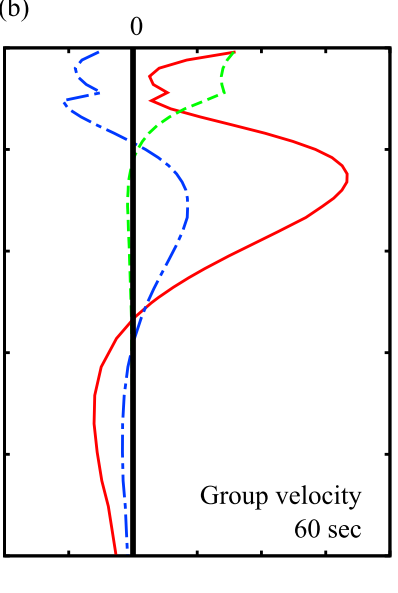

(c)

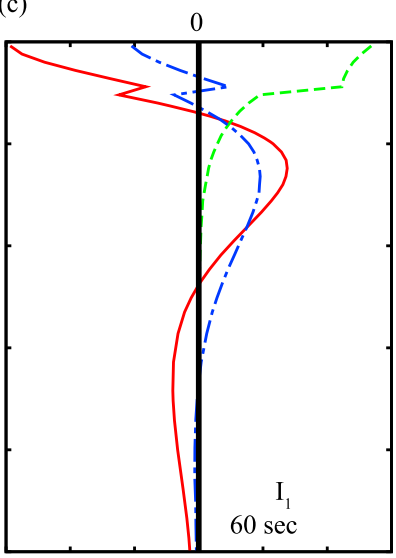

(g)

(f)

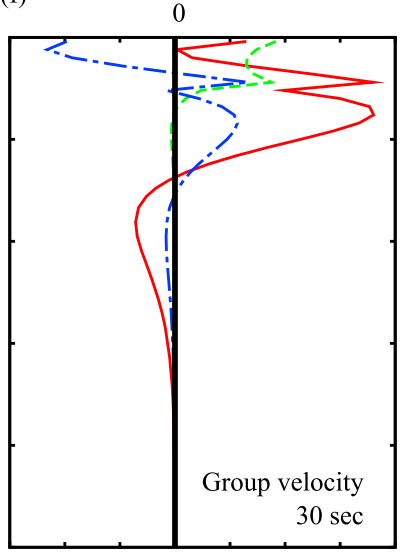

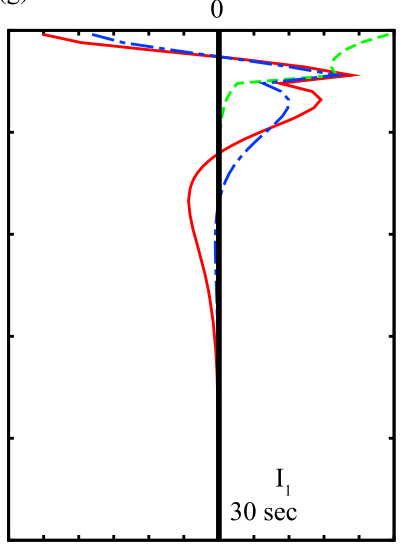

(d)

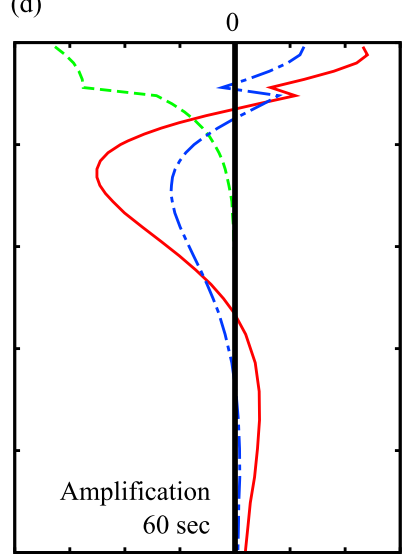

(h)

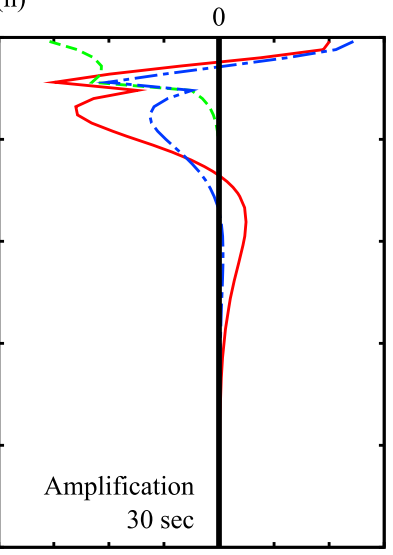

Figure 12. (a-d) The depth sensitivity kernels of phase velocity, group velocity, the $\mathrm{I}_{1}$ integral, and amplification for the $60 \mathrm{~s}$ Rayleigh wave due to shear velocity (Vs; solid red line), compressional velocity (Vp; green dashed line), and density ( $\rho$; blue dot dashed line) perturbations. (e-h) Same as Figures 12a-12d except for the $30 \mathrm{~s}$ Rayleigh wave.

southeast is located southeast of the significant amplitude increase for waves heading northwest. Assuming that these features are solely due to the amplification effect across the velocity structure boundary and the 2-D approximation discussed in section 2 is correct, we would expect them to be nearly exactly colocated in the two maps (Figures $5 \mathrm{c}$ and $5 \mathrm{f}$ ). The apparent shift of these features as a function of the direction of wave propagation potentially represents the breakdown of the 2-D approximation and suggests leakage of the amplification effect into the observed attenuation structure. In fact, Yang and Forsyth [2006b] have shown through a numerical simulation that the amplification effect due to a sharp structural boundary only gradually approaches its 2-D theoretical prediction after the simulated Rayleigh wave passes the boundary. Because the amplification effect is much larger than the attenuation effect for local measurements, the details of this apparent shift need to be understood before high-resolution attenuation tomography can be performed reliably.

\section{Structural Sensitivity of Local Amplification}

[37] In this section we compare the depth sensitivity kernels of local amplification with other types of 2-D surface wave quantities such as phase velocity, group velocity, and the $I_{1}$ integral defined in equation (4). The $I_{1}$ quantity is included here because it can be estimated based on equation (3) when phase velocity, group velocity, and amplification have all been measured locally. Here we use the 1-D PREM earth model [Dziewonski and Anderson, 1981] as the reference model and compute the depth sensitivity kernels numerically by perturbing the model. The 2-D surface wave quantities for any given 1-D model are calculated using the code developed by Herrmann and Ammon [2004]. Based on equation (3), the depth sensitivity of the amplification can also be expressed as

$$
\frac{\delta \beta}{\beta}=-\frac{1}{2}\left(\frac{\delta c}{c}+\frac{\delta C}{C}+\frac{\delta I_{1}}{I_{1}}\right),
$$

where the depth sensitivity of phase velocity c, group velocity $\mathrm{C}$, and the $\mathrm{I}_{1}$ integral can be further expressed by the perturbation of $\mathrm{Vs}, \mathrm{Vp}$, and density structure [Zhou et al., 2004].

[38] Figure 12 summarizes the sensitivity kernels for the $30 \mathrm{~s}$ and $60 \mathrm{~s}$ Rayleigh waves due to perturbations in compressional velocity (Vp), shear velocity (Vs), and density $(\rho)$. While the surface wave attenuation $(1 / \mathrm{Q})$ kernels are not 
shown here, they are very similar to the phase velocity kernels by replacing the shear and compressional velocity perturbations (Vs and $\mathrm{Vp}$ ) by shear and compressional anelastic perturbations (1/Qs and 1/Qp), respectively [Dalton et al., 2008].

[39] The phase and group velocities, which are the observables for most traditional surface wave tomography, are clearly dominantly sensitive to shear velocity (Vs) structure with longer period Rayleigh waves more sensitive to deeper structure (Figures 12a and $12 \mathrm{~b}$ and Figures 12e and 12f). While velocity measurements are also affected by $\mathrm{Vp}$ and density, the generally weaker sensitivity and potential tradeoff often prevent them from being estimated simultaneously with Vs. The $I_{1}$ integral and the local amplification sensitivity kernels, on the other hand, are different from phase and group velocity sensitivity kernels in a few distinguishing ways (Figures $12 \mathrm{c}$ and $12 \mathrm{~d}$ and Figures $12 \mathrm{~g}$ and $12 \mathrm{~h}$ ). First, unlike phase and group velocities, the sensitivities to $\mathrm{Vp}$ and $\mathrm{Vs}$ perturbations have opposite signs at shallow depths. While a fast $\mathrm{Vp}$ perturbation near the surface reduces the amplification, a fast Vs perturbation intensifies the amplification effect. Due to this opposing effect, the shallow $\mathrm{Vp} / \mathrm{Vs}$ ratio may potentially be better studied based on amplification measurements. Second, the density structure has a relatively stronger effect on amplification than on phase and group velocities. This is particularly true for the quantity $I_{1}$ (Figures $12 \mathrm{c}$ and $12 \mathrm{~g}$ ) when both phase and group velocity can be independently constrained. Hence, we believe that the ability to constrain local amplification across a wide range of frequencies along with dispersive phase and group velocity measurements will be the key to studying 3-D density structure. Third, while the local amplification is still strongly affected by shear wave perturbations, the amplification kernels due to shear wave perturbations, unlike phase and group velocity kernels, have three zero crossings. This makes the local amplification more sensitive to rapid shear velocity variations with depth than group and phase velocities. Due to the broad depth sensitivity kernel, traditional 1-D inversion methods based on phase and group velocity dispersion curves alone often suffer from the tradeoff between structures at nearby depths. This tradeoff and the nonuniqueness of the inversion problem may potentially be mitigated by jointly inverting the period dependence of both phase and group velocities and amplification measurements.

[40] While the ability to determine local amplification promises to provide new constraints on 3-D variations in crustal and upper mantle structure, it should be remembered that the local amplification determined based on our method is only a relative quantity. The fact that the mean amplification over an area is arbitrary means that the observed amplification cannot be used to invert independently for 1-D structure at each location. The comparison between the observed and predicted amplifications is only meaningful when lateral variations are considered (e.g., Figures 8 and 9).

[41] With Rayleigh waves sensitive to compressional velocity, shear velocity, and density, it is desirable to have three independent types of measurements to resolve the model tradeoffs and nonuniqueness. Because the group velocity dispersion, in theory, can be derived from the phase velocity dispersion, the phase velocity and amplification alone only provide two independent types of constraints. The H/V ratio [e.g., Tanimoto and Rivera, 2008], or ellipticity, of Rayleigh waves, which describes the amplitude ratio between radial $(\mathrm{H})$ and vertical $(\mathrm{V})$ component particle motion, is another independent 2-D quantity that potentially may be used in a joint inversion. Initial attempts to determine Rayleigh wave H/V ratios across USArray have shown clear correlations with known geological features in the western U.S. (F. C. Lin et al., Joint inversion of Rayleigh wave phase velocity and ellipticity using USArray: Constraining velocity and density structure in the upper crust, submitted to Journal of Geophysical Research, 2012). Unlike Rayleigh waves, however, Love waves are only sensitive to shear velocity and density structure and the inversion based on Love wave velocity and amplification measurements may avoid the trade-off due to variations in compressional velocity. While Rayleigh and Love waves are primarily sensitive to vertically and horizontally polarized shear waves, respectively, which can be different in the presence of radial anisotropy [Moschetti et al., 2010a], they are both sensitive to the same density structure.

\section{Conclusions}

[42] In this study we present an empirical method to construct surface wave amplification and large-scale attenuation maps across a large array using the phase travel time and amplitude maps observed across the array. The method utilizes spatial differential operators to first determine the local amplitude variation and the focusing/defocusing correction and then separates the amplification and attenuation effects by examining the directional dependence of the amplitude decay measurements.

[43] The ability to construct surface wave amplification maps may lead to not only new and unique constraints on 3-D velocity and density structures but also smaller bias in phase velocity and attenuation measurements. Partly due to the traditional sparse station configuration of seismic networks and perhaps partly due to the presumed larger uncertainties in amplitude instrument responses, surface wave amplification to constrain earth structure has been extremely rare. Here, we demonstrate that the amplification maps that we construct across USArray not only correlate well with known major tectonic features across the western U.S. but also compare well quantitatively with predictions based on new 3-D velocity models [e.g., Lin et al., 2011a]. There are discrepancies, however, between the observed and predicted amplifications, which may indicate errors in the 3-D models. While the errors may be in the velocity model, density model, or more likely both, the discrepancies can be mitigated by constructing a more realistic density model. In particular, we show that without a density jump between the crust and mantle across the Moho, the predicted amplification cannot explain the $30 \mathrm{~s}$ Rayleigh wave amplification in regions such as the Southern Rockies and the Columbia Basin where the crust is anomalously thick or thin.

[44] Large-scale attenuation maps, which clearly separates the highly attenuative tectonically active western U.S. from the less attenuative stable cratonic eastern U.S., are probably most useful to study long-wavelength crustal and upper mantle temperatures. For the $30 \mathrm{~s}$ Rayleigh wave, the observed attenuation also agrees well with several known geological features assuming strong attenuation is observed in regions characterized by high temperatures. Detailed attenuation structure at longer periods may be more biased 


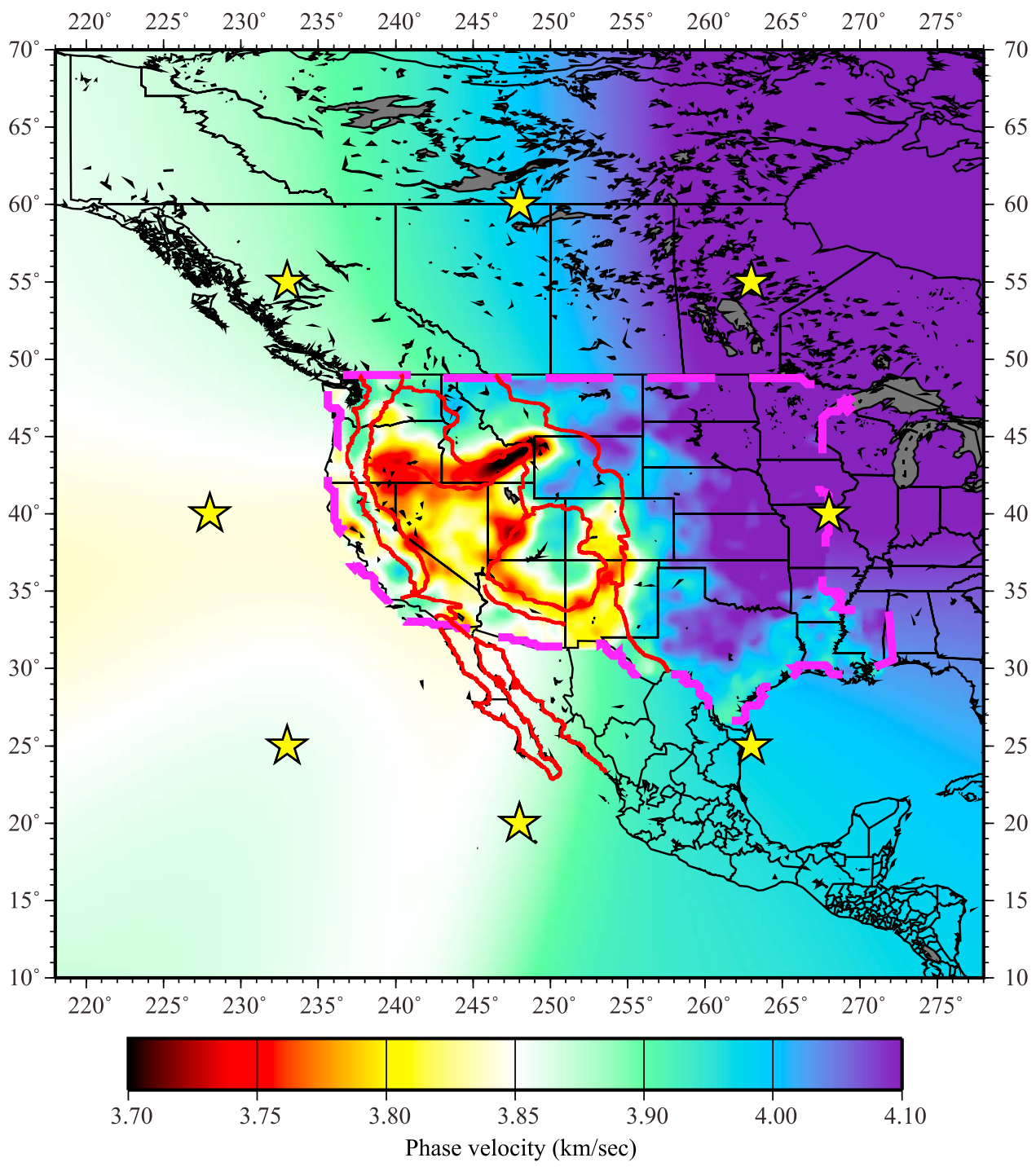

Figure A1. The reference phase velocity model used in the synthetic experiment. Within the pink dashed line, the phase velocity map is equal to the map estimated by Helmholtz tomography [Lin and Ritzwoller, 2011a]. The map is smoothly extrapolated to cover the entire simulation area outside the dashed line. The eight stars mark the source locations of the 2-D simulations.

because of the smaller effect of anelastic attenuation on the observed amplitude variations compared to other effects such as amplification and focusing/defocusing from elastic inhomogeneities. While the effects of detailed 3-D wave phenomena on amplification and attenuation measurements remain to be understood, we anticipate that this will be resolved based on numerical studies in the not too distant future.

[45] The natural extension of this study is to perform a 3-D velocity, density, and anelasticity inversion based on the observed phase velocity, amplification, and attenuation measurements across a broad range of periods. To obtain a better constraint on crustal structure, the amplification and attenuation information within the microseism period band $(5-20 \mathrm{~s})$ is desired. One of the technical challenges is to develop reliable means to extract amplitude information from ambient noise cross correlations [Tsai, 2011; Lin et al., 2011b]. We are optimistic that this development, along with the potential for a joint inversion involving $\mathrm{H} / \mathrm{V}$ ratio of Rayleigh waves, Love wave measurements, and body wave measurements, will allow for increasingly detailed and accurate crustal and upper mantle structures to be resolved.

\section{Appendix A: 2-D Synthetic Test}

[46] In this section, we use the 2-D finite difference method to simulate $60 \mathrm{~s}$ Rayleigh waves coming from eight different directions (Figure A1) and test the method for constructing amplification maps that we present here. For simplicity, we perform the simulations in Cartesian coordinates where $1^{\circ}$ longitude and latitude are both set equal to $100 \mathrm{~km}$. For each simulation, we solve the 2-D wave equation for an inhomogeneous medium:

$$
\frac{\partial^{2} u_{2 D}(\mathbf{r}, t)}{\partial t^{2}}=\nabla \cdot\left[c^{2}(\mathbf{r}) \nabla u_{2 D}(\mathbf{r}, t)\right]
$$


(a)

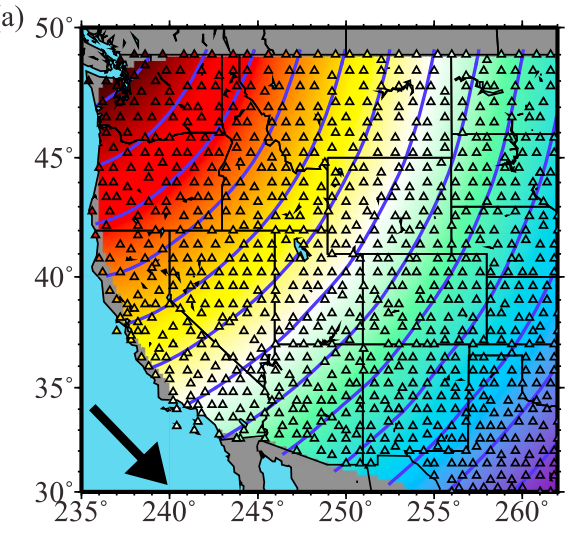

(a) 50
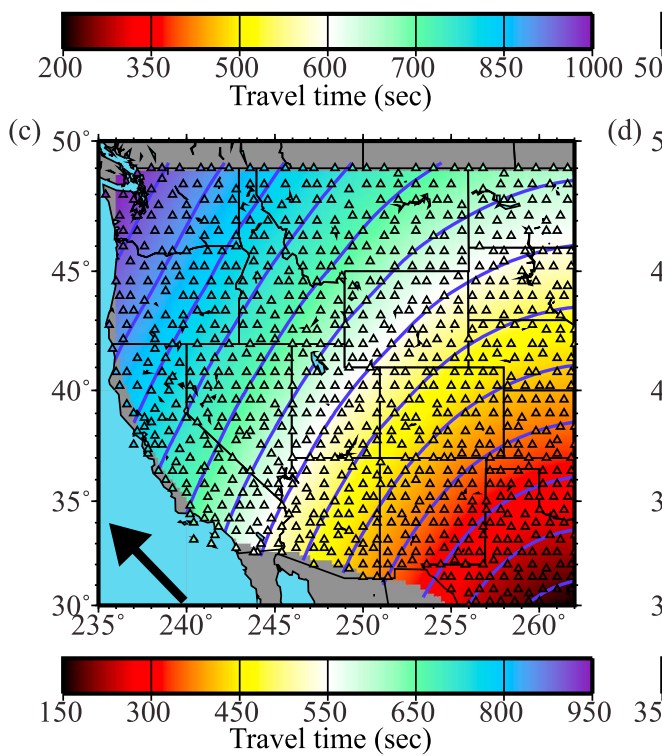

(b) 50

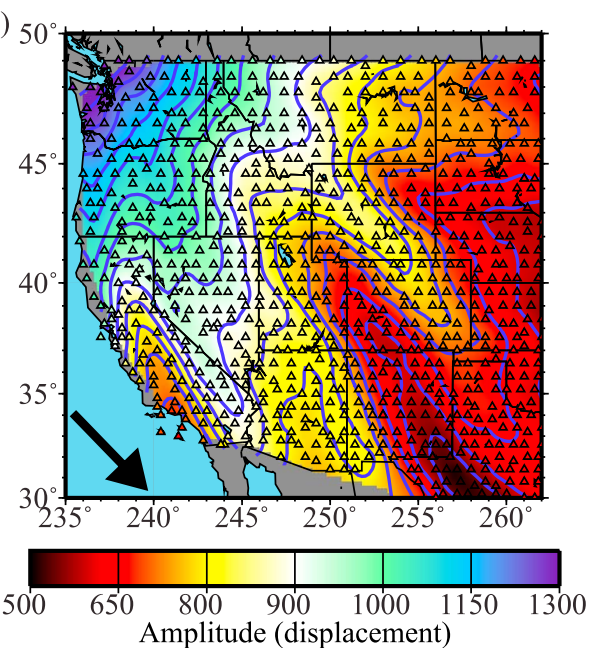

(d) 50

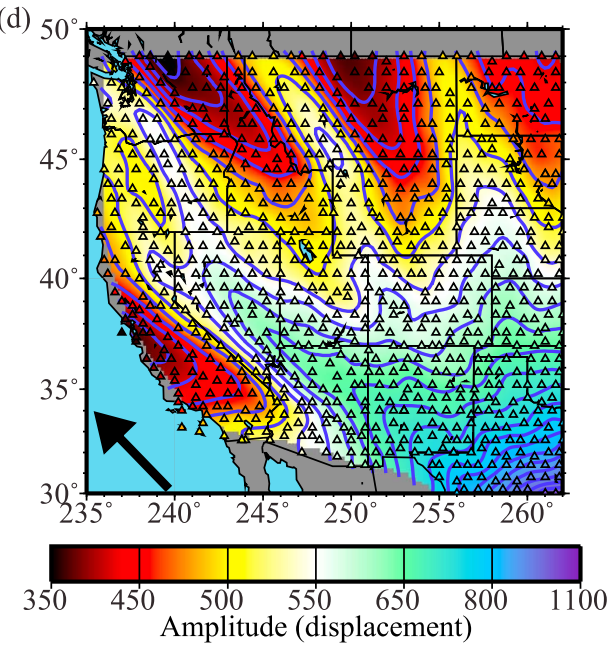

Figure A2. $(a, b)$ Same as Figures $2 a$ and $2 b$ except that the results derive from the synthetic measurements for a source in the northwestern direction. (c, d) Same as Figures A2a and A2b except for a source in the southeastern direction. The amplitude contours are separated by intervals of 30 and 50 displacement units in Figures A2b and A2d, respectively.

where $c$ is the phase speed and $c^{2}$ can be considered to be the effective shear modulus (with a constant density equal to 1 ). We introduce no attenuation in order to focus exclusively on the amplification effect. While the 2-D simulation does not fully account for 3-D surface wave propagation and will not reproduce the empirical observations, the simulation does result in semirealistic amplification due to the conservation of 2-D energy flux. We use the $60 \mathrm{~s}$ Rayleigh wave phase velocity map from Helmholtz tomography derived by Lin and Ritzwoller [2011a] as the reference model where smoothing is applied outside of the array to cover the entire simulation area (Figure A1). To obtain a single frequency wave, we use a harmonic oscillating source in each simulation and obtain both synthetic phase travel time and synthetic amplitude measurements across the array. Figure A2 shows an example of synthetic phase travel time and amplitude maps constructed for two simulations with waves propagating in the southeast and northwest directions.

[47] Similar to the derivation shown in section 2, if we substitute the single frequency expression of the 2-D wavefield, $u_{2 D}=A \exp [i \omega(t-\tau)]$, into equation (A1), the imaginary part of the solution can be expressed as

$$
\frac{2 \nabla(1 / c) \cdot \nabla \tau}{(1 / c)}=\frac{2 \nabla A \cdot \nabla \tau}{A}+\nabla^{2} \tau .
$$

Equation (A2) can be directly compared with equation (8). We see that the role of the local amplification $\beta$ is played in this simulation by the inverse of phase velocity $c$.

[48] We apply the same processes as described in section 3 to account for focusing/defocusing and construct the amplification map based on the synthetic measurements. Figure A3 shows examples of the apparent amplitude decay (first term on the RHS of equation (A2)), the focusing/defocusing correction term (second term on the RHS of equation (A2)), and the corrected amplitude decay (the entire RHS of equation (A2)) derived from the synthetic measurements shown in Figure A2 for waves coming from the northwest and southeast directions. Similar to the results shown in Figure 5, clear anticorrelations can again be observed between the apparent 

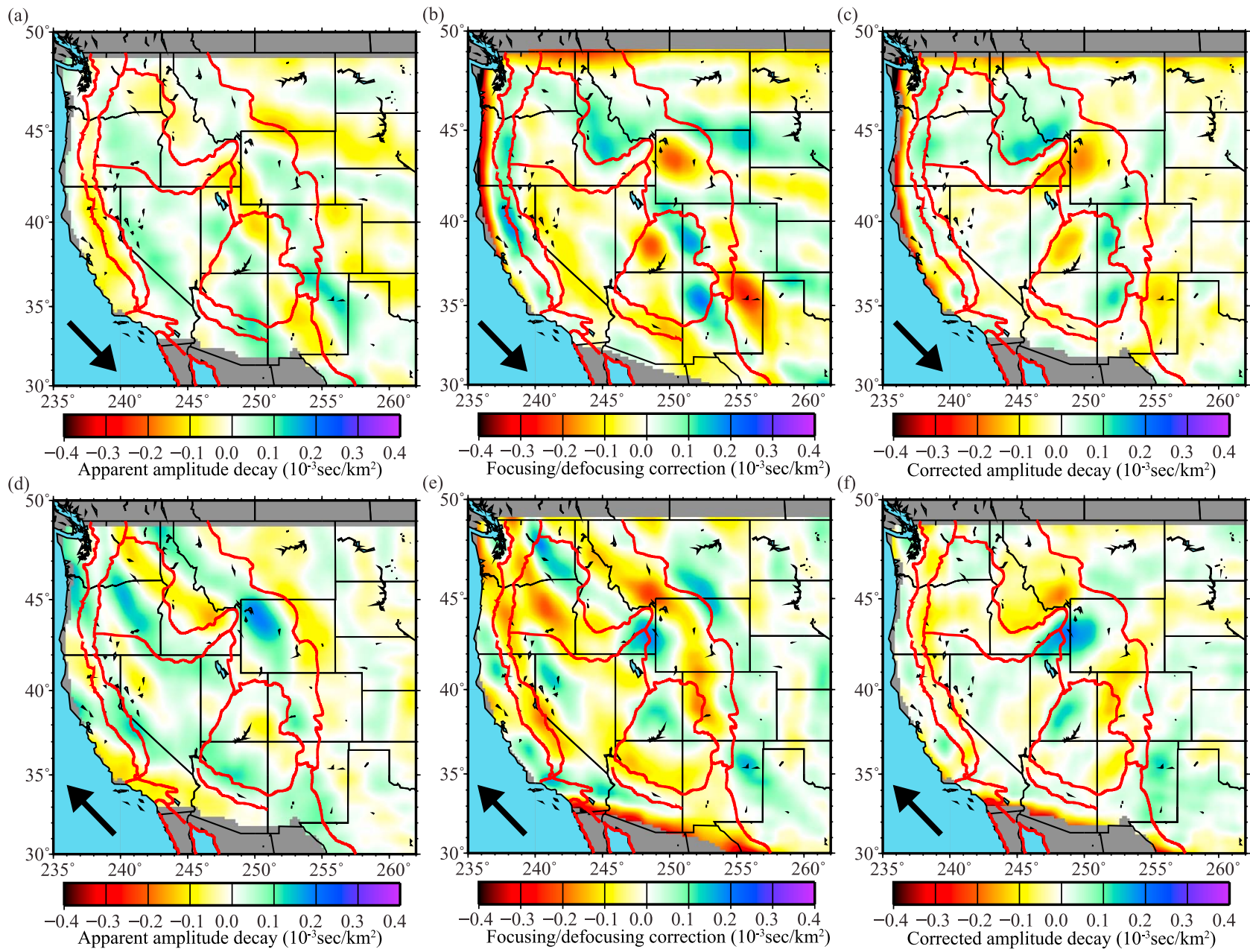

Figure A3. Same as Figure 5 except for the synthetic data set.

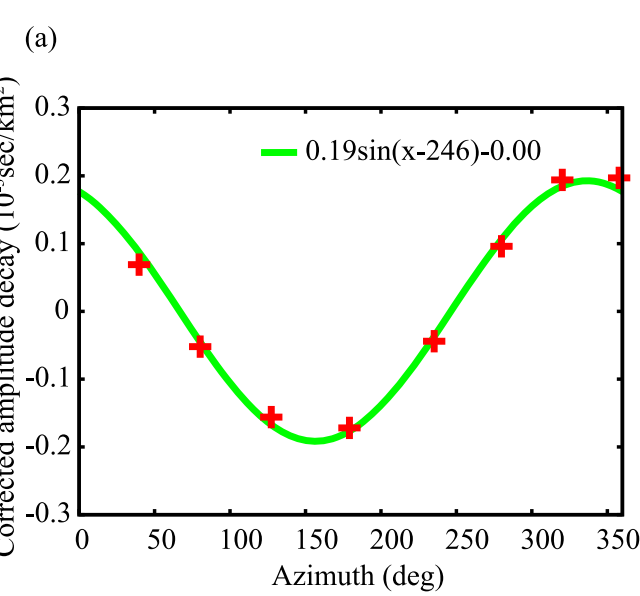

(b)

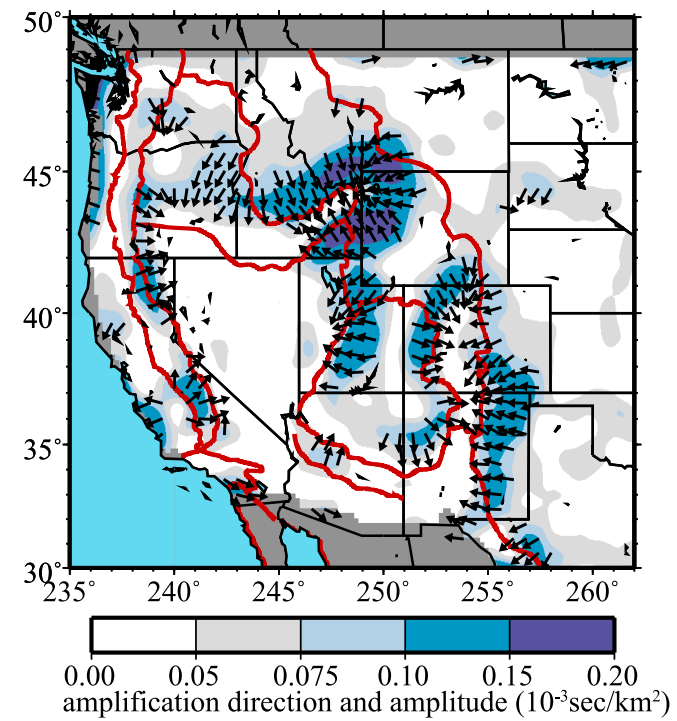

Figure A4. Same as Figures $6 \mathrm{~b}$ and $6 \mathrm{c}$ except for the synthetic data set. 
(a)

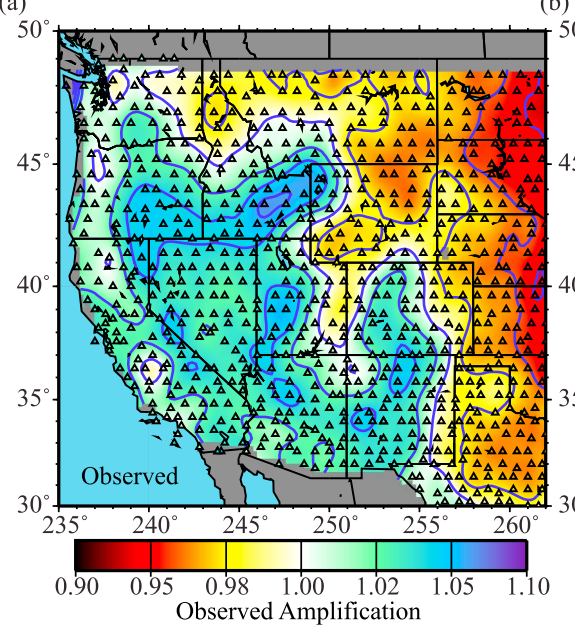

(b)

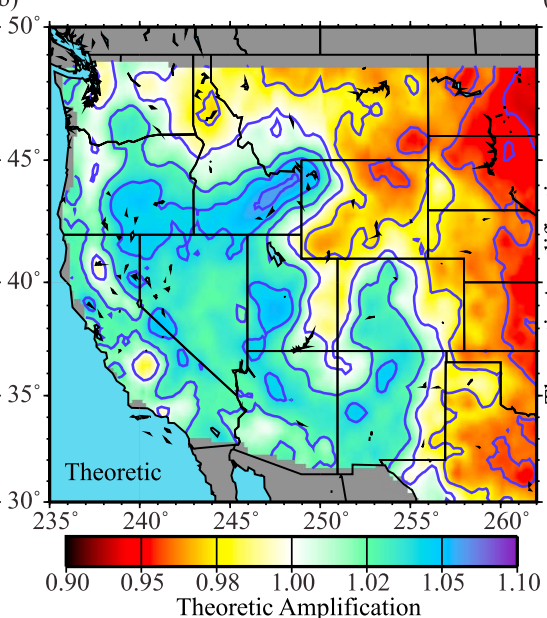

(c)

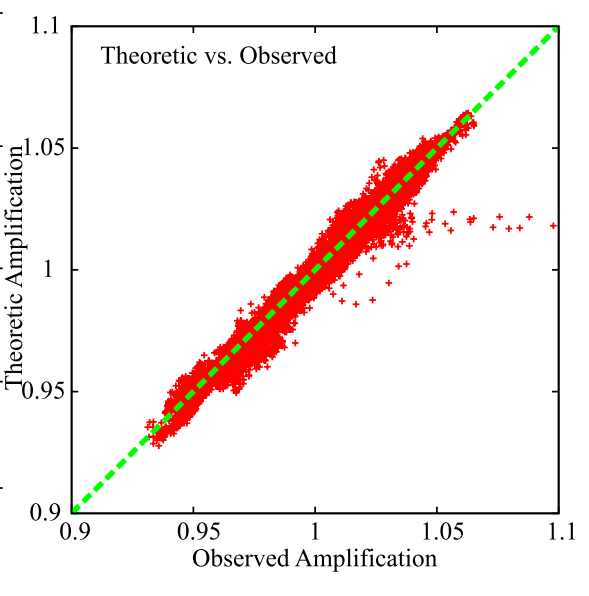

Figure A5. (a) Same as Figure 7c except for the synthetic data set. (b) The theoretical amplification $\mathrm{c}^{\prime} / \mathrm{c}$ calculated based on the reference $60 \mathrm{~s}$ Rayleigh wave phase velocity map where $\mathrm{c}^{\prime}$ is the reciprocal of the average slowness $1 / \mathrm{c}$ in the area. (c) The relationship between the theoretical and observed amplification shown in Figures A5a and A5b where each point represents the result at a single grid point on the maps. The green dashed line is the reference line for complete agreement.

amplitude decay and focusing/defocusing correction maps for both of the directions (Figures $\mathrm{A} 3 \mathrm{a}$ and $\mathrm{A} 3 \mathrm{~b}$ and Figures $\mathrm{A} 3 \mathrm{~d}$ and $\mathrm{A} 3 \mathrm{e}$ ). Clear anticorrelation can also be observed in the corrected amplitude decay maps for waves propagating in the two opposite directions (Figures A $3 \mathrm{c}$ and A3f). The pattern of corrected amplitude decay maps (Figures A3c and A3f) generally agrees with the empirical results (Figures 5c and 5f), although now with a smaller variation in amplitude. Note that because we do not consider attenuation in our simulation, the corrected amplitude decay is solely due to the amplification effect (equation (A2)). To estimate the maximum amplification direction and amplitude, we again examine the directionally dependent corrected amplitude decay measurements (e.g., Figure A4a). The observed maximum amplification directions and amplitudes for the whole region are summarized in Figure A4b. The inverted amplification map after three iterations is shown in Figure A5a, which now can be directly compared with the theoretical prediction based on our input reference phase velocity map (Figures A5b and A5c).

[49] Overall, the constructed amplification map agrees well with the theoretical amplification map. A cluster of discrepant points is observed in Figure A5c with larger than predicted amplifications (observed $>1.03$; predicted $<1.03$ ). These points are mostly located near the northwestern corner of the maps. The wavefield is not well constrained near the edge of the station coverage, which results in higher uncertainty in the observed amplification. The fact that the majority of the observed amplification is within $1 \%$ of the theoretical prediction demonstrates the accuracy and robustness of the method.

[50] Acknowledgments. The authors wish to thank two anonymous reviewers for constructive comments and B. Schmandt and R. W. Clayton for helpful discussions. The facilities of the IRIS Data Management System, specifically the IRIS Data Management Center, were used to access the waveform and metadata required in this study. The IRIS DMS is funded through the National Science Foundation and specifically the GEO Directorate through the Instrumentation and Facilities Program of the National
Science Foundation under Cooperative Agreement EAR-0552316. This research was supported by the Director's Post Doctoral Fellowship of the Seismological Laboratory at the California Institute of Technology and by NSF grants EAR-0711526 and EAR-0844097 at the University of Colorado Boulder.

\section{References}

Dalton, C. A., and G. Ekström (2006a), Constraints on global maps of phase velocity from surface-wave amplitudes, Geophys. J. Int., 167, 820-826, doi:10.1111/j.1365-246X.2006.03142.x.

Dalton, C. A., and G. Ekström (2006b), Global models of surface wave attenuation, J. Geophys. Res., 111, B05317, doi:10.1029/2005JB003997.

Dalton, C. A., G. Ekström, and A. M. Dziewoński (2008), The global attenuation structure of the upper mantle, J. Geophys. Res., 113, B09303, doi:10.1029/2007JB005429.

Dziewonski, A. M., and D. L. Anderson (1981), Preliminary reference Earth model, Phys. Earth Planet. Inter., 25, 297-356, doi:10.1016/ 0031-9201(81)90046-7.

Ekström, G., J. Tromp, and E. W. F. Larson (1997), Measurements and global models of surface wave propagation, J. Geophys. Res., 102, 8137-8157, doi:10.1029/96JB03729.

Gilbert, H. (2012), Crustal structure and signatures of recent tectonism as influenced by ancient terranes in the western United States, Geosphere, 8, 141-157, doi:10.1130/GES00720.1.

Herrmann, R. B., and C. J. Ammon (2004), Computer programs in seismology: Surface waves, receiver functions and crustal structure, manual, St. Louis Univ., St. Louis, Mo.

Lawrence, J. F., and G. A. Prieto (2011), Attenuation tomography of the western United States from ambient seismic noise, J. Geophys. Res., 116, B06302, doi:10.1029/2010JB007836.

Levshin, A. L., X. Yang, M. P. Barmin, and M. H. Ritzwoller (2010), Midperiod Rayleigh wave attenuation model for Asia, Geochem. Geophys. Geosyst., 11, Q08017, doi:10.1029/2010GC003164.

Liang, C., and C. A. Langston (2009), Wave gradiometry for USArray: Rayleigh waves, J. Geophys. Res., 114, B02308, doi:10.1029/ 2008JB005918.

Lin, F., and M. H. Ritzwoller (2011a), Helmholtz surface wave tomography for isotropic and azimuthally anisotropic structure, Geophys. J. Int., 186, 1104-1120, doi:10.1111/j.1365-246X.2011.05070.x.

Lin, F., and M. H. Ritzwoller (2011b), Apparent anisotropy in inhomogeneous isotropic media, Geophys. J. Int., 186, 1205-1219, doi:10.1111/ j.1365-246X.2011.05100.x.

Lin, F., M. P. Moschetti, and M. H. Ritzwoller (2008), Surface wave tomography of the western United States from ambient seismic noise: Rayleigh and Love wave phase velocity maps, Geophys. J. Int., 173(1), 281-298, doi:10.1111/j.1365-246X.2008.03720.x. 
Lin, F., M. H. Ritzwoller, and R. Snieder (2009), Eikonal tomography: Surface wave tomography by phase front tracking across a regional broad-band seismic array, Geophys. J. Int., 177(3), 1091-1110, doi:10.1111/j.1365-246X.2009.04105.x.

Lin, F., M. H. Ritzwoller, Y. Yang, M. P. Moschetti, and M. J. Fouch (2011a), Complex and variable crustal and uppermost mantle seismic anisotropy in the western United States, Nat. Geosci., 4, 55-61, doi:10.1038/ngeo1036.

Lin, F., M. H. Ritzwoller, and W. Shen (2011b), On the reliability of attenuation measurements from ambient noise cross-correlations, Geophys. Res. Lett., 38, L11303, doi:10.1029/2011GL047366.

Lowry, A. R., N. M. Ribe, and R. B. Smith (2000), Dynamic elevation of the Cordillera, western United States, J. Geophys. Res., 105(B10), 23,371-23,390, doi:10.1029/2000JB900182.

Jackson, I., J. Fitz Gerald, U. Faul, and B. H. Tan (2002), Grain-sizesensitive seismic wave attenuation in polycrystalline olivine, J. Geophys. Res., 107(B12), 2360, doi:10.1029/2001JB001225.

Maceira, M., and C. J. Ammon (2009), Joint inversion of surface wave velocity and gravity observations and its application to central Asian basins shear velocity structure, J. Geophys. Res., 114, B02314, doi:10.1029/2007JB005157.

Moschetti, M. P., M. H. Ritzwoller, F. C. Lin, and Y. Yang (2010a), Seismic evidence for western-US deep-crustal deformation caused by extension, Nature, 464, 885-889, doi:10.1038/nature08951.

Moschetti, M. P., M. H. Ritzwoller, F.-C. Lin, and Y. Yang (2010b), Crustal shear wave velocity structure of the western United States inferred from ambient seismic noise and earthquake data, J. Geophys. Res., 115, B10306, doi:10.1029/2010JB007448.

Pollitz, F. F. (2008), Observations and interpretation of fundamental mode Rayleigh wavefields recorded by the Transportable Array (USArray), J. Geophys. Res., 113, B10311, doi:10.1029/2007JB005556.

Pollitz, F. F., and J. A. Snoke (2010), Rayleigh-wave phase-velocity maps and three-dimensional shear velocity structure of the western US from local non-plane surface wave tomography, Geophys. J. Int., 180 1153-1169, doi:10.1111/j.1365-246X.2009.04441.x.

Prieto, G. A., J. F. Lawrence, and G. C. Beroza (2009), Anelastic Earth structure from the coherency of the ambient seismic field, J. Geophys. Res., 114, B07303, doi:10.1029/2008JB006067.

Shapiro, N. M., M. Campillo, L. Stehly, and M. H. Ritzwoller (2005), High resolution surface wave tomography from ambient seismic noise, Science, 307(5715), 1615-1618, doi:10.1126/science.1108339.

Smith, W. H. F., and P. Wessel (1990), Gridding with continuous curvature splines in tension, Geophysics, 55, 293-305, doi:10.1190/1.1442837.
Tanimoto, T. (1990), Modelling curved surface wave paths: Membrane surface wave synthetics, Geophys. J. Int., 102, 89-100, doi:10.1111/j.1365246X.1990.tb00532.x.

Tanimoto, T. (1991), Waveform inversion for three-dimensional density and $S$ wave structure, J. Geophys. Res., 96(B5), 8167-8189, doi:10.1029/ 91JB00196.

Tanimoto, T., and L. Rivera (2008), The $\mathrm{ZH}$ ratio method for long-period seismic data: Sensitivity kernels and observational techniques, Geophys. J. Int., 172, 187-198, doi:10.1111/j.1365-246X.2007.03609.x.

Trampert, J., and J. H. Woodhouse (1996), High resolution global phase velocity distributions, Geophys. Res. Lett., 23, 21-24, doi:10.1029/ 95GL03391.

Tromp, J., and F. A. Dahlen (1992), Variational principles for surface wave propagation on a laterally heterogeneous Earth-II. Frequency-domain JWKB theory, Geophys. J. Int., 109, 599-619, doi:10.1111/j.1365-246X. 1992.tb00120.x.

Tromp, J., and F. A. Dahlen (1993), Variational principles for surface wave propagation on a laterally heterogeneous Earth-III. Potential representation, Geophys. J. Int., 112, 195-209, doi:10.1111/j.1365-246X.1993. tb01449.x.

Tsai, V. C. (2011), Understanding the amplitudes of noise correlation measurements, J. Geophys. Res., 116, B09311, doi:10.1029/2011JB008483.

Wielandt, E. (1993), Propagation and structural interpretation of non-plane waves, Geophys. J. Int., 113, 45-53, doi:10.1111/j.1365-246X.1993. tb02527.x.

Yang, Y., and D. Forsyth (2006a), Rayleigh wave phase velocities, smallscale convection, and azimuthal anisotropy beneath southern California, J. Geophys. Res., 111, B07306, doi:10.1029/2005JB004180.

Yang, Y., and D. Forsyth (2006b), Regional tomographic inversion of the amplitude and phase of Rayleigh waves with 2-D sensitivity kernels, Geophys. J. Int., 166, 1148-1160, doi:10.1111/j.1365-246X.2006. 02972.x.

Yang, Y., and D. Forsyth (2008), Attenuation in the upper mantle beneath southern California: Physical state of the lithosphere and asthenosphere, J. Geophys. Res., 113, B03308, doi:10.1029/2007JB005118.

Yang, Y., M. H. Ritzwoller, F. C. Lin, M. P. Moschetti, and N. M. Shapiro (2008), Structure of the crust and uppermost mantle beneath the western United States revealed by ambient noise and earthquake tomography, J. Geophys. Res., 113, B12310, doi:10.1029/2008JB005833.

Zhou, Y., F. A. Dahlen, and G. Nolet (2004), Three-dimensional sensitivity kernels for surface wave observables, Geophys. J. Int., 158, 142-168, doi:10.1111/j.1365-246X.2004.02324.x. 